\title{
Properties of $\mathrm{OH} / \mathrm{IR}$ stars with IRAS LRS spectra
}

\author{
P. S. Chen ${ }^{1,2,3}$, R. Szczerba ${ }^{3,2}$, S. Kwok ${ }^{2}$, and K. Volk ${ }^{2}$ \\ 1 Yunnan Observatory and United Laboratory of Optical Astronomy, CAS, Kunming 650011, China \\ 2 Department of Physics and Astronomy, University of Calgary, Calgary, Alberta T2N 1N4, Canada \\ 3 N. Copernicus Astronomical Center, Rabiańska 8, 87-100 Toruń, Poland
}

Received 31 January 2000 / Accepted 11 January 2001

\begin{abstract}
OH}$ satellite line $(1612 \mathrm{MHz})$ maser sources associated with the IRAS counterparts were assembled from the literature. Cross-correlation between collected OH/IR - IRAS sources and the recent catalogue of the IRAS LRS spectra (Kwok et al. 1997) allows us to subdivide 1024 of them (after excluding OH sources with uncertain IRAS associations) into 10 groups according to the Volk \& Cohen (1989) classification scheme. We have found that sources with silicate emission form the largest group (about 57\%), followed by the group with silicate absorption (only about 16\%). For more sensitive individual $\mathrm{OH}$ maser surveys these numbers differentiate even more and the ratio between silicate emission and absorption sources reaches a value close to 6 . Surprisingly, we found an association of $\mathrm{OH}$ maser emission with a small number of stars with carbon-rich atmospheres. The distribution of these subgroups of $\mathrm{OH} / \mathrm{IR}$ stars in the IRAS colour-colour and the period-colour diagrams as well as histograms of some physical properties are presented. The galactic distribution of important parameters (periods, IRAS colours, expansion and stellar velocities) for different classes of OH/IR sources is analyzed from the point of view of the stars' evolutionary status. The correlations among the periods, colours, expansion and star velocities and the pumping efficiencies for selected subgroups of $\mathrm{OH} / \mathrm{IR}$ stars are also investigated. These analyses allow us to demonstrate that a significant fraction of silicate emission group sources do not form an evolutionary sequence with the silicate absorption objects - as is suggested e.g. by the IRAS colour-colour diagram, but rather form a lower initial mass sequence, i.e. these sources will never develop sufficient mass loss to manifest silicate absorption.
\end{abstract}

Key words. masers - astronomical data bases: miscellaneous - stars: AGB and post-AGB (stars:) circumstellar matter

\section{Introduction}

The study of stellar maser sources, especially $\mathrm{OH}$ maser sources is important in astronomy since it provides an intense, collimated beacon that can serve as a probe of physical conditions for the sources in which $\mathrm{OH}$ maser emission has been generated. The high luminosity of the $\mathrm{OH}$ masers, their weak interaction with the ambient medium and transparency to interstellar reddening, allows for their detection at great distances. Some of the source properties can be inferred even without modeling of the maser process itself (Elitzur 1992). In such cases the stellar maser radiation provides a general tool to study and to examine many fundamental problems in astrophysics including: distance measurements, properties of the Galaxy (e.g. galactic rotation and galactic magnetic field), interstellar scattering, the structure of circumstellar mat-

Send offprint requests to: R. Szczerba,

e-mail: szczerba@ncac.torun.pl ter and the galactic distribution and kinematics of the Asymptotic Giant Branch (AGB) stars.

Following the first detection of astronomical masers by Weaver et al. (1965) and the discovery of the $\mathrm{OH}$ maser emission from circumstellar shells of four infrared (IR) late-type stars by Wilson \& Barrett (1968), investigations in this field developed very rapidly. The characteristic double peaked $1612 \mathrm{MHz} \mathrm{OH}$ emission was identified to result from an expanding envelope (Kwok 1976), and an increasing number of $\mathrm{OH}$ sources were detected towards many optically identified stars but also towards objects with only IR counterparts or even without any counterpart. The maser sources related to the sources with IR excess soon acquired the name of $\mathrm{OH} / \mathrm{IR}$ stars. Among $\mathrm{OH} / \mathrm{IR}$ sources the most numerous group is a group of type II OH/IR stars (see e.g. Wilson \& Barret 1972) which shows stronger satellite line emission at $1612 \mathrm{MHz}$ than main-line emission at 1665 and $1667 \mathrm{MHz}$. It is generally accepted that majority of $\mathrm{OH} / \mathrm{IR}$ stars are highly 
evolved, low or intermediate mass AGB stars (initial mass smaller than about $\left.6-8 M_{\odot}\right)$. They are characterized by a very wide range of mass loss rates from about $10^{-7}$ up to $10^{-4} M_{\odot} \mathrm{yr}^{-1}$ which leads in the extreme case to the formation of an optically thick dust shell around the source (e.g. Baud \& Habing 1983). These stars are likely to be the progenitors of planetary nebulae (Herman \& Habing 1985; Kwok 1987).

The comparatively high detection rate of $\mathrm{OH}$ emission among sources observed by Wilson \& Barrett (1972) was the motivation for the more systematic searches of the sky on regular grids. Prior to the IRAS mission in 1983, such systematic $1612 \mathrm{MHz} \mathrm{OH}$ emission surveys near the galactic plane revealed a moderate number of $\mathrm{OH} / \mathrm{IR}$ stars (e.g. Caswell \& Haynes 1975; Winnberg et al. 1975; Johansson et al. 1977a, 1977b; Bowers 1978a, 1978b; Baud et al. 1979a, 1979b; Caswell et al. 1981; Olnon et al. 1981; Habing et al. 1983). A complete data base of the pre-IRAS observations has been compiled by te Lintel Hekkert et al. (1989; hereafter tLVHW) with a total of 442 objects, of which only a few are without associations with IRAS sources. Since most of the energy of OH/IR stars is emitted by the circumstellar dust between 2 and $60 \mu \mathrm{m}$ (e.g. Kleinmann et al. 1981), a very successful approach in finding new $\mathrm{OH} / \mathrm{IR}$ stars was to search the IRAS Point Source Catalogue (1988, hereafter PSC) for sources with colours resembling those of the AGB stars with oxygen-rich envelops (see e.g. Olnon et al. 1984). Several systematic surveys have been performed to detect $\mathrm{OH}$ emission from sources selected according to their colours: the Arecibo (hereafter AO) surveys (Eder et al. 1988; Lewis et al. 1990; Lewis 1992a; Chengalur et al. 1993; Lewis 1994); the Dutch-Parkes (hereafter tL-H) surveys (te Lintel Hekkert 1990; te Lintel Hekkert et al. 1991); the Nançay surveys (Sivagnanam et al. 1990; Le Squeren et al. 1992; David et al. 1993; te Lintel Hekkert \& Chapman 1996) and others (e.g. Gaylard et al. 1989; Galt et al. 1989; Likkel 1989; te Lintel Hekkert 1991; Silva et al. 1993). At the same time, the "blind" $\mathrm{OH}$ surveys especially in the galactic plane and the galactic center were continued. They include: VLA surveys (Zijlstra et al. 1989; Lindqvist et al. 1992; Blommaert et al. 1994) and the ATCA/VLA surveys (Sevenster et al. 1997a, 1997b). The investigations of a representative sample of mostly nearby Mira stars by Sivagnanam et al. $(1988,1989)$ should be mentioned as well. The intensive $\mathrm{OH}$ emission surveys cited above and the availability of photometric and spectroscopic data from the IRAS sky survey have made possible a comprehensive study of the physical properties and evolutionary status for $\mathrm{OH} / \mathrm{IR}$ stars.

In this paper, we have assembled from the literature a general sample of $\mathrm{OH} / \mathrm{IR}$ stars, as large as possible, that have associations with the IRAS sources. The IRAS Low Resolution Spectra (LRS) of these $\mathrm{OH} / \mathrm{IR}$ stars are examined and the nature of the circumstellar dust classified. The distributions of these subgroups of $\mathrm{OH} / \mathrm{IR}$ stars in the IRAS colour-colour diagram and the period--colour diagram are presented. The galactic and the velocity dis- tributions of these subgroups of $\mathrm{OH} / \mathrm{IR}$ stars are investigated to understand their evolutionary status. The correlations among the periods, colours, expansion and stellar velocities and the pumping efficiencies for different subgroups of $\mathrm{OH} / \mathrm{IR}$ stars are analyzed to outline differences and similarities between their physical properties.

\section{Working sample of $\mathrm{OH} / \mathrm{IR}$ stars}

The first comprehensive catalogue of $\mathrm{OH} / \mathrm{IR}$ stars with $1612 \mathrm{MHz}$ emission is the tLVHW catalogue which includes 442 objects observed before the IRAS mission. The infrared counterparts on the basis of positional information from the IRAS PSC are given for 437 out of 442 objects, but for 165 sources the difference between IRAS and $\mathrm{OH}$ positions is larger than $1^{\prime}$. This could be related to the fact that a large fraction of $\mathrm{OH}$ sources detected in single dish surveys do not have accurate positions but also that a significant number of them are located in the IRAS confused area (see tLVHW). Lewis (1992b) compiled an extensive list that contained the $\mathrm{OH} / \mathrm{IR}$ stars detected mainly in $1612 \mathrm{MHz}$ maser emission prior to 1992. For convenience it is named here as the Lewis Catalogue, in which all pre-IRAS detections from tLVHW, most AO detections, most objects from the Dutch-Parkes survey, most of the Nançay detections and most detections from the literature before 1992 are included. In the Lewis Catalogue there are $1522 \mathrm{OH} / \mathrm{IR}$ stars with IRAS associations. Of these, 1515 were detected in the $\mathrm{OH} 1612 \mathrm{MHz}$ maser line. Note, however, that the attributed associations are not always certain (see below).

We have searched the available literature to find new $\mathrm{OH} / \mathrm{IR}$ sources which were detected after the Lewis Catalogue. We have also checked the Lewis (1992b) bibliography to include any additional sources. In some cases (Dickinson \& Chaisson 1973; Engels 1979; Sivagnanam et al. 1988; van Langevelde et al. 1992; Lindqvist et al. 1992; Ivison et al. 1994; Sjouwerman et al. 1998) we have attributed IRAS associations to and included only such objects for which the $\mathrm{OH}$ position differs by less than $1^{\prime}$ from the position of the nearest IRAS PSC source. In consequence, we have included a further $343 \mathrm{OH} / \mathrm{IR}$ sources with IRAS associations to give a total number of $1858 \mathrm{OH} / \mathrm{IR}$ stars with IRAS identification. These sources have been cross-correlated with the recently published catalogue for the IRAS LRS spectra (Kwok et al. 1997) which contains 11224 spectra classified by eye according to the scheme of Volk \& Cohen (1989). Out of 1858 entries, 1065 sources have LRS spectra available and they compose our working sample of $\mathrm{OH} / \mathrm{IR}$ stars in this paper.

All $1065 \mathrm{OH} / \mathrm{IR}$ stars with the IRAS LRS spectra are listed in Table $1^{1}$. The sources are grouped by the University of Calgary LRS classification scheme using

\footnotetext{
1 Table 1 is only available in electronic form at the CDS via anonymous ftp to cdsarc.u-strasbg.fr (130.79.128.5) or via

http://cdsweb.u-strasbg.fr/cgi-bin/qcat?J/A+A/368/1006
} 
the letter codes defined by Volk \& Cohen (1989) and ordered by right ascension within each group. The last two columns of Table 1 contain information about the quality of the IRAS association and references for the $\mathrm{OH}$ maser observations (the entry on the first position in the reference list shows the preferred data source). If the quality of association is denoted by " 1 " it means that the difference between $\mathrm{OH}$ maser and IRAS PSC positions is larger than $1^{\prime}$ and such sources are not taken into account for later analysis. In our catalogue we have found 27 such sources in the tLVHW compilation and 14 in the Australia Telescope Compact Array (ATCA) survey (Sevenster et al. 1997a, 1997b). In consequence, only 1024 sources were used for the investigation of statistical properties of $\mathrm{OH} / \mathrm{IR}$ stars in this paper.

Among $1024 \mathrm{OH} / \mathrm{IR}$ stars with the LRS spectra, 588 are in group E (silicate emission feature at $9.7 \mu \mathrm{m}$ and/or at $18 \mu \mathrm{m}$, mostly equivalent to the LRS number classification $2 \mathrm{n}$ ), 163 in group A (silicate absorption feature at $9.7 \mu \mathrm{m}$, equivalent to the LRS number classification $3 \mathrm{n}$ ), 60 in group $\mathrm{H}$ (red-continuum, as well as either $9.7 \mu \mathrm{m}$ silicate absorption feature or the $11.3 \mu \mathrm{m}$ PAH feature, mostly equivalent to the number classification $7 \mathrm{n}$ ), 32 in group P (spectra with relatively red continua from 13 to $23 \mu \mathrm{m}$ and a sharp rise at the blue end of the spectrum or else show the 11.3 or $12.5 \mu \mathrm{m}$ PAH molecular features, mostly equivalent to the LRS number classification 8n), 28 in group $\mathrm{F}$ (featureless dust continuum flatter than that expected of a stellar atmosphere, mostly equivalent to the LRS number classification 10-16), 9 in group C (showing $11.3 \mu \mathrm{m}$ SiC emission), 1 in group $\mathrm{S}$ (stellar photospheric continuum), 2 in group $\mathrm{L}$ (showing spectrum with cool continuum and the $12.8 \mu \mathrm{m}$ [Ne II] line — see Volk \& Cohen 1990), 75 in group U (unusual spectrum) and finally 66 in group I (noisy or incomplete spectrum).

With an attempt to make our sample uniform we have taken the $\mathrm{OH}$ maser related data preferably from: tL-H (462); AO (274); tLVHW (114) and ATCA surveys (69 sources with IRAS association better than $1^{\prime}$ ) which composes about $90 \%$ of the analyzed sample. Data for an additional $105 \mathrm{OH} / \mathrm{IR}$ sources were taken from more than 20 papers. A complete bibliographical list together with the abbreviations used is attached to the end of Table 1.

\section{Data analysis and discussion}

In this section the nature of the $\mathrm{OH} / \mathrm{IR}$ sources from our sample of 1024 sources in different LRS groups is investigated on the basis of their infrared colours, variabilities, periods, galactic distributions, flux densities in the infrared and the $1612 \mathrm{MHz}$, envelope and stellar velocities.

\subsection{Circumstellar features of $\mathrm{OH} / \mathrm{IR}$ stars}

In contrast to the early beliefs that OH/IR stars represent the very last stage of AGB evolution and therefore are associated with oxygen-rich AGB stars with silicate absorption features, we found $57.4 \%$ of $\mathrm{OH}$ sources in our sample are associated with silicate emission (E) objects, followed by silicate absorption (A, 15.9\%). A more careful inspection of our data in Table 1 shows that our finding seems to not be related to the sample selection. The four most important surveys (tl-H, AO, tLVHW and ATCA) provide about $90 \%$ of data for these two groups with the relative numbers (counting detection in each survey, independently) between $\mathrm{E}$ and A sources: $284 / 92(=3.07), 186 / 31(=6), 91 / 62(=1.47)$ and $30 / 5(=6)$, respectively. Note that the ratio of 3.61 for the whole sample compiled by us is close to the ratio of 3.07 for the IRAS colour-selected Dutch-Parkes (tL-H) survey. Since the $\mathrm{OH}$ emission of $\mathrm{E}$ type maser sources is usually weaker than A type ones it becomes clear that the blind preIRAS observations (tLVHW), which had rather low sensitivity, tend to show relatively low ratio (1.47) between $\mathrm{E}$ and A objects. On the other hand, the higher sensitivity surveys (about 0.03 Jy) AO (IRAS colour selected sample) and ATCA (blind observations of galactic bulge and galactic disc) both detected 6 times more (!) E group objects than A group sources. The change of the silicate feature from emission to absorption is related to the increase of optical depth inside the circumstellar envelope, and this has been interpreted as increasing mass loss rate sequence for the AGB stars (e.g. Bedijn 1987; Volk \& Kwok 1988; van der Veen \& Habing 1988). However, Volk \& Kwok (1987) pointed out that the relative population and galactic distribution of emission and absorption objects suggests that the absorption objects originate from a higher mass main-sequence population and that not all emission sources evolve into absorption ones. This very important statement will be further investigated in Sect. 3.3 and 3.4 using our larger sample.

A small number of $\mathrm{OH} / \mathrm{IR}$ stars are in group $\mathrm{H}$ (5.9\%). As Volk \& Cohen (1989) pointed out, these sources should be either planetary nebulae $(\mathrm{PNe})$, reflection nebulae, or HII region sources. It is generally accepted that at the end of the AGB phase, the $\mathrm{OH} / \mathrm{IR}$ star will stop pulsating and consequently the large scale mass loss rate will cease as well. The $\mathrm{OH} / \mathrm{IR}$ star will then evolve through the proto-planetary nebula $(\mathrm{PPN})$ stage to become a planetary nebula (e.g. Kwok 1987; van der Veen et al. 1987). After the star has moved off the AGB phase the $1612 \mathrm{MHz} \mathrm{OH}$ maser line will remain observable for a period of about 100-1000 yr until the $\mathrm{OH}$ column density becomes too low for the maser to be saturated (Sun \& Kwok 1987). Indeed, we have found that $17 \mathrm{OH} / \mathrm{IR}$ stars (about 28\%) in group $\mathrm{H}$ are identified as $\mathrm{PPNe}$ or $\mathrm{PNe}$ (these are indicated by an asterisk at the last position in the corresponding row of Table 1). $\mathrm{OH}$ maser emission is also expected during a short period in the life of a compact HiI regions (e.g. te Lintel Hekkert 1991; Elitzur 1992). Of the group H objects IRAS 17439-2845, IRAS 18092-1742, IRAS 19343+2926, IRAS $20255+3712$ and IRAS $22176+6303$ are Sharpless HII regions; IRAS 17417-2851 is an ultra-compact HII region and IRAS 17574-2403 belongs to W 28. 


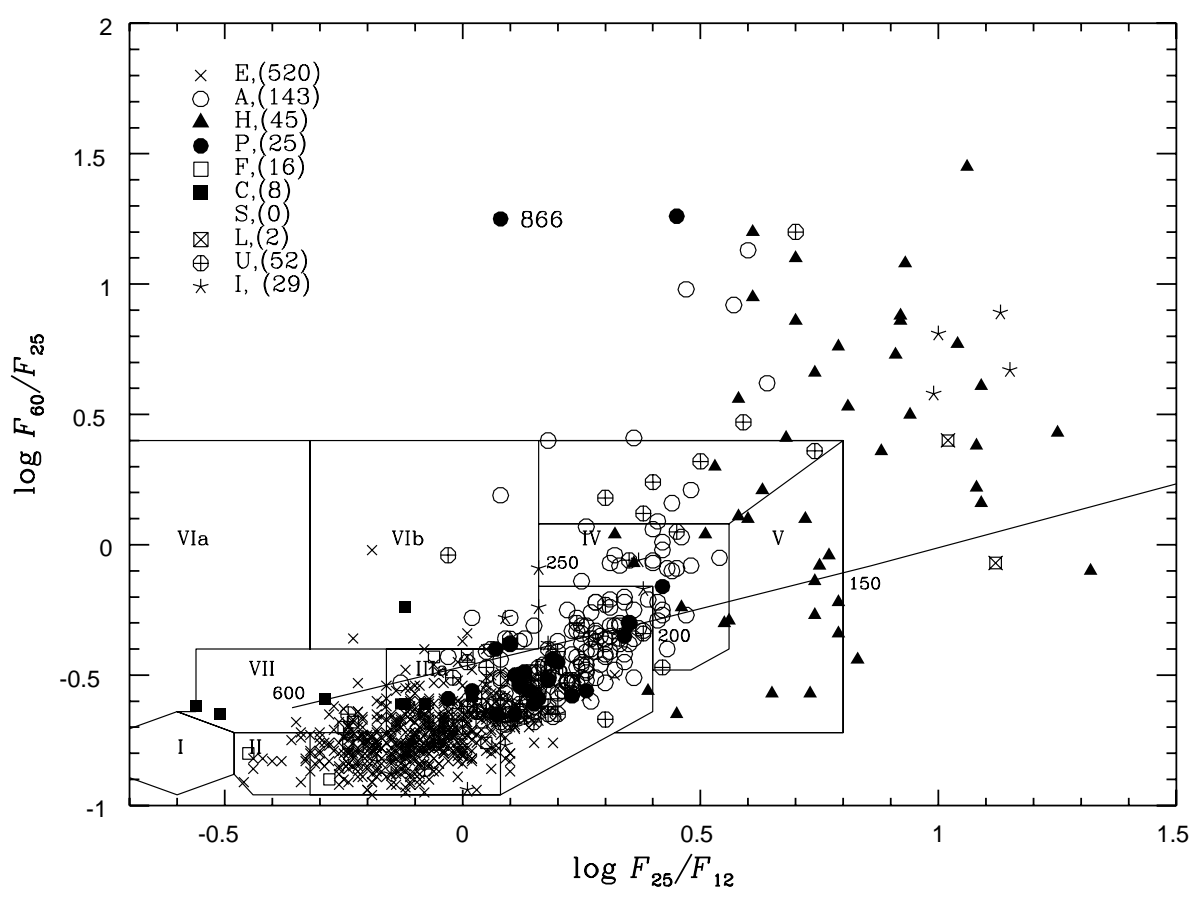

Fig. 1. IRAS colour-colour diagram for OH/IR stars in different LRS groups. Symbols used are described inside the plot together with total number of sources plotted in each group in parentheses. One ultra-compact HII region IRAS $20149+3440$ is identified by means of its number (866) from Table 1 . Solid line shows the behaviour of the blackbody temperature with 4 values $(600,250,200$ and $150 \mathrm{~K})$ shown at the proper position in $\log F_{25} / F_{12}$

An even smaller number of $\mathrm{OH} / \mathrm{IR}$ stars is found in group $\mathrm{F}(2.7 \%)$ with featureless spectra. The status of these objects is not clear because these sources are expected to be of two populations: sources with very low dust optical depth and those with optical depth large enough for the silicate feature to be intermediate between emission and absorption (Volk \& Kwok 1988). It is also of interest that Omont et al. (1993) have argued that some sources with featureless spectra, especially those with LRS numbers 11-12 are very cool carbon-rich stars rather than the oxygen-rich ones.

Since almost all carbon for an oxygen-rich star is locked in $\mathrm{CO}$ molecules, it is surprising that we found $9 \mathrm{OH} / \mathrm{IR}$ stars with LRS spectra which classify them as $\mathrm{C}$ group objects on the basis of having $11.3 \mu \mathrm{m} \mathrm{SiC}$. Among them there are 3 sources (IRAS 04130+3918, IRAS 05373-0810 and IRAS 18551+0323) with associations in the CCGCS catalogue (Stephenson 1989). In addition, IRAS 17371-3021 and IRAS $18551+0323$ are thought to be genuinely carbon-rich (Le Squeren et al. 1992; Volk et al. 1992). On the other hand, Zijlstra et al. (1991) argued that IRAS 08304-4313 is possibly not carbon-rich due to confusion.

Another group of stars with probable carbon related chemistry is group P (3.1\%). Spectra of some sources in group $\mathrm{P}$ are very similar to silicate absorption spectra, while some other sources are Population I WolfRayet stars (Volk \& Cohen 1989). Sources in Table 1 with Wolf-Rayet type spectra include: IRAS 17459-3057, IRAS 19327+3024 in group P (as the central star of a PN),
IRAS 18204-1344 in group E (as a companion of a symbiotic star) and IRAS 07027-7934 in group H (as the central star of a PN).

A moderate number of $\mathrm{OH} / \mathrm{IR}$ stars belong to group $\mathrm{U}(7.3 \%)$. The nature of most of these sources is unknown (Volk \& Cohen 1989). However, some of the sources in group $\mathrm{U}$ are the best candidates of the extreme carbon stars (Volk et al. 1992). All sources with presence of both (carbon- and oxygen-based) types of circumstellar chemistry (if proven) need a special attention. We will discuss AGB and/or post-AGB sources with C, H, P and F spectra in the forthcoming papers.

Note that there is one source (IRAS 18548+0637) in group S. This source has $\log \left(F_{25} / F_{12}\right)=-0.39$ (where $F_{\lambda}$ is the PSC flux at wavelength $\lambda$ in Jy) and is located on the borderline between group $\mathrm{S}$ and group $\mathrm{F}$ in the LRS data base, which means that the source has some circumstellar material. On the other hand, the SES1 flag equal to 0000 in all IRAS photometric bands suggests that no extended sources are associated with it. In the HST Guide Star Catalogue (1989, hereafter HST GSC), there are two visible stars with magnitude in $\mathrm{V}$ of $9.2(\mathrm{~V} 840 \mathrm{Aql})$ and 13.0 within the beam size of the Arecibo Telescope (about $3^{\prime}$ ) that was used to observe IRAS $18548+0637$. Within $3^{\prime}$ radius from the position of $\mathrm{V} 840 \mathrm{Aql}$ there is no other IRAS source. It is not clear whether the $\mathrm{OH}$ maser originates in the envelope of V $840 \mathrm{Aql}$ or is produced around another relatively faint star. 


\subsection{The IRAS colour-colour diagram of $\mathrm{OH} / \mathrm{IR}$ stars}

The IRAS colour--colour diagram has long been proved to be an efficient and simple tool for discussion of properties and evolutionary status of AGB stars (e.g., Olnon et al. 1984; Zuckerman \& Dyck 1986; Volk \& Kwok 1988; van der Veen \& Habing 1988; Kwok 1990). The $\log \left(F_{60} / F_{25}\right)$ versus $\log \left(F_{25} / F_{12}\right)$ colour-colour diagram for all $\mathrm{OH} / \mathrm{IR}$ stars with the IRAS LRS spectra and good quality $(Q=3$ or 2$)$ IRAS PSC fluxes is presented in Fig. 1. For reference, the regions defined by van der Veen \& Habing (1988) are shown. We did not try to exclude known HiI regions from our sample. For example, one such object (IRAS 20149+3440) with an extreme position on this diagram is marked by means of its number (866) from Table 1. From a statistical point of view, however, most of the $\mathrm{OH} / \mathrm{IR}$ sources shown on the colour-colour diagram follow the well known behavior established for oxygen-rich AGB stars. This has been interpreted as continuous increasing mass loss rates ranging from about $10^{-7} M_{\odot} \mathrm{yr}^{-1}$ at the lower left part of group E sources up to $10^{-4} M_{\odot} \mathrm{yr}^{-1}$ at the upper right part of the group A objects (Bedijn 1987; Volk \& Kwok 1988). As a consequence of increasing mass loss rate the silicate feature changes from emission to the absorption due to increase in optical depth (Volk \& Kwok 1988) and the infrared colour increases and the colour temperature declines. Our Fig. 1 implies that $\mathrm{OH}$ maser activity is common during the AGB and post-AGB phase and all groups of objects can show this kind of activity.

Sources in group E form a very tight group in the lower left corner of the diagram with colour temperatures around 600-300 K; sources in group A are quite well separated from these in group $\mathrm{E}$ at $\log \left(F_{25} / F_{12}\right)=0.1$ and occupy an area to the upper right of the group E with colour temperatures around $300-180 \mathrm{~K}$. Note that the group E sources with $\log \left(F_{25} / F_{12}\right)<0$, in general, are known to have the properties of Mira variables (e.g. van der Veen \& Habing 1988). Sources in group $H$ are found in the area to the upper right of the group A objects with wide range of both colours but in general with lower temperature $(<180 \mathrm{~K})$. Between groups $\mathrm{A}$ and $\mathrm{H}$ there is also a clear separation around $\log \left(F_{25} / F_{12}\right)=0.5$. During the transition from the AGB to the PPNe and then to PNe (transition from objects with A type LRS spectra to the $\mathrm{H}$ type LRS spectra) the circumstellar dust shell moves away from the central star and cools down, so more and more energy will be radiated in the far-IR and colour temperature will decrease (e.g. Bedijn 1987; Kwok 1987; te Lintel Hekkert 1990). Zijlstra et al. (1989) pointed out that the large spread in colour for PPNe and PNe could be due to some geometrical effects, different dust density and content in the envelopes, and different radiation fields around the objects.

The sources from group $\mathrm{P}$ are mainly located in between group $\mathrm{E}$ and $\mathrm{A}$, and this may indicate that at least some of them are misclassified group A sources. It is clearly seen also that among 8 group $\mathrm{C}$ sources plotted in
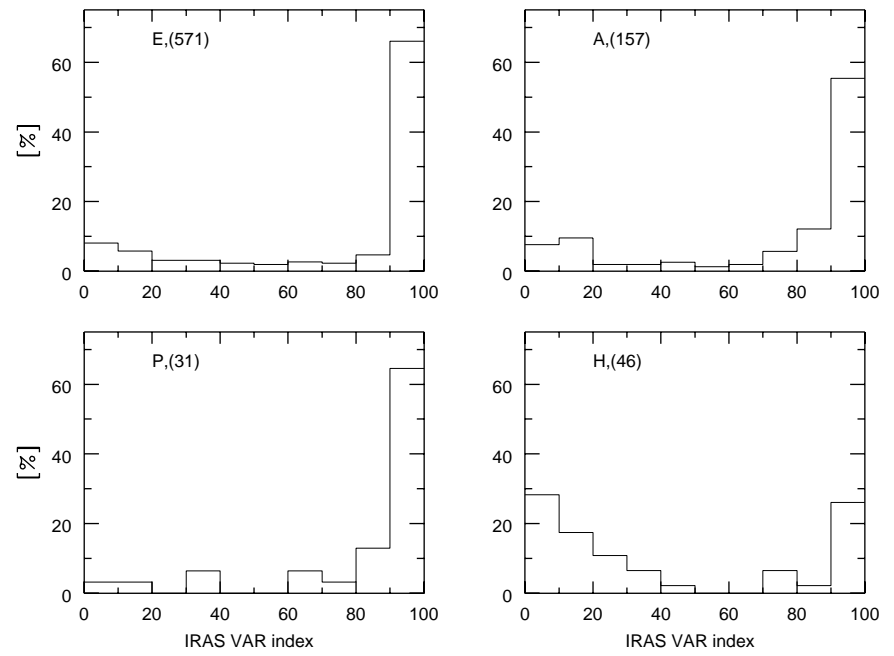

Fig. 2. The histograms of the probability of variability for $\mathrm{OH} / \mathrm{IR}$ stars in groups E, A, P and H. Numbers in parentheses show how many sources in each group have been used to construct the histogram

Fig. 1 some of them are located in the visual carbon star region (VII), and the remainder are in the infrared or extreme carbon star regions (Chan \& Kwok 1988, 1990; Volk et al. 1992) which overlap with the positions of $\mathrm{P}$ stars.

Most of the sources in group $F$ are located in the group E region. In spite of the general properties of this group (see Sect. 3.1) among objects selected here on the basis of the $\mathrm{OH}$ maser presence there seems to be almost no objects with intermediate optical depth, i.e. objects located in between group $\mathrm{E}$ and $\mathrm{A}$. As will be discussed further, some of group F objects share other common physical properties with sources of group E.

The scenario discussed above is based on the presence of a definite colour sequence passing from $\mathrm{OH} / \mathrm{IR}$ stars in group E to group A then to group $\mathrm{H}$ in the IRAS colourcolour diagram. This tendency could be interpreted as an evolutionary sequence of the $\mathrm{OH} / \mathrm{IR}$ stars evolving from those with emission features to these with absorption features, then to the planetary nebula phase. In this scenario, mass loss continuously increases with time of evolution and given star occupies successively the regions of the group E, A, and finally of PPNe and PNe (group H) where the heavy mass loss ceases. On the other hand, Volk \& Kwok (1987), Likkel (1989) and Sivagnanam \& Le Squeren (1990) have argued that the observed colour sequence could depend also on the initial mass of the star, in such a sense that only the more massive sources can attain the reddest colours. Probably, both these tendencies play a role but it is impossible to distinguish between them solely using the IRAS two-colour diagram.

\subsection{Variability and period of $\mathrm{OH} / \mathrm{IR}$ stars}

During the IRAS mission in total three surveys were made. After comparison of data from these three surveys, each IRAS source was given a "variability number" (hereafter 
VAR) ranging from 1 to 99 to indicate the probability of variability in the infrared. The larger the number, the larger the probability that the source is variable in the infrared. In general, if VAR $>80$, a source is considered to be a variable star (van der Veen \& Habing 1988).

The histograms of the VAR distributions for $\mathrm{OH} / \mathrm{IR}$ stars from our sample (sources with $\mathrm{VAR}=0$ or -1 have not been included) in groups $\mathrm{E}, \mathrm{A}, \mathrm{P}$ and $\mathrm{H}$ are presented in Fig. 2. It is obvious that sources in groups E, $\mathrm{A}$ and $\mathrm{P}$ have very high probabilities of variability, while sources in group $\mathrm{H}$ have much lower ones. Percentages of the sources with VAR $>80$ are $71 \%, 68 \%$ and $76 \%$ for groups E, A and P, respectively, and only about $30 \%$ for group $\mathrm{H}$. Note that PPNe and PNe from Table 1 which belong to group $\mathrm{H}$ have VAR $<40$ with the only exception of IRAS 07399-1435 with VAR = 99. Since mass loss from AGB stars is driven by stellar pulsations, the variability is related to mass loss process (e.g. van der Veen et al. 1987). Therefore $\mathrm{OH} / \mathrm{IR}$ stars in groups $\mathrm{E}, \mathrm{A}$ and $\mathrm{P}$ are undergoing mass loss whereas most sources in group $\mathrm{H}$ may have stopped losing mass according to the infrared variability shown here.

The period, $P$, versus colour diagrams of $\mathrm{OH} / \mathrm{IR}$ stars from our sample are plotted in Fig. 3 at the top panel for colour $\log \left(F_{25} / F_{12}\right)$ and in the bottom panel for $\log \left(F_{60} / F_{25}\right)$. All sources in Table 1 that have "good" IRAS association, known period and good quality fluxes are included in this figure. Periods have been taken from Herman \& Habing (1985); Kholopov et al. (1985-88); Whitelock et al. (1994); Wood et al. (1998). Numbers plotted near the group name shows total number of sources used. Some sources which do not follow the general trend are identified by their numbers from Table 1 . Three of them are PPNe and 2 are ultra-compact HiI regions. The sources in group E have periods which range between several hundred and 1000 days with a mean of approximately 440 days, and sources in group A have periods from about 500 to over 2000 days with a mean around 1150 days. It is clearly seen that for group $\mathrm{E}$ sources the periods of $\mathrm{OH} / \mathrm{IR}$ stars increase the infrared colour $\log \left(F_{25} / F_{12}\right)$ increases as well, while for group A sources the correlation between period and colour essentially breaks down. For the same colour we can find periods with very different values (in general, larger than in group E). Since especially $\log \left(F_{25} / F_{12}\right)$ colour is a measure of the optical depth (mass loss rate) for oxygen-rich AGB sources (e.g. van der Veen \& Habing 1988), then the above correlation for group $\mathrm{E}$ objects shows that their mass loss rate is a strong function of the pulsational period. Pulsational period is expected to increase when mass of the star decreases but also when a star ascends the AGB due to an increase in its radius (Kwok 1987). In consequence, the increase of pulsational period with colour for group E objects is to some degree due to an evolutionary sequence. However, as in the colour-colour diagram a possible initial mass dependence may be hidden in the period--colour diagrams.
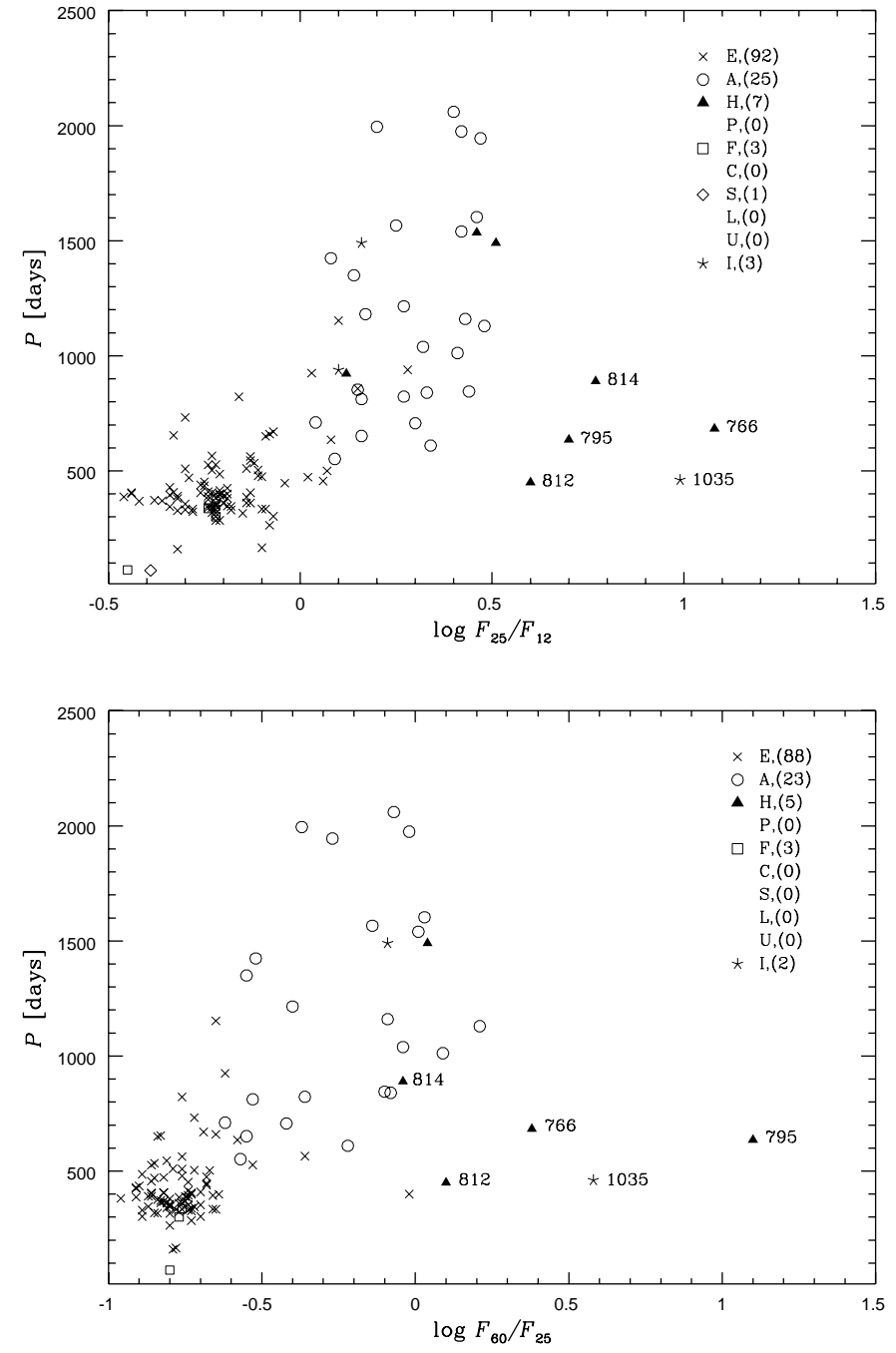

Fig. 3. The $P$-colour diagrams of $\mathrm{OH} / \mathrm{IR}$ stars from our sample: Total number of sources plotted in each group is shown in parentheses

In an attempt to separate these two effects in Fig. 4 we have plotted the galactic latitude $(|b|)$ as a function of pulsational period for The top panel of Fig. 4 shows that our sample splits into two subgroups: one at high galactic latitude $\left(|b|>10^{\circ}\right)$ composed mainly of $E$ type sources (note that $3 \mathrm{~F}$ type objects, for which we have $P$, belong to this subgroup) with period not exceeding about 700 days and the second one dominated by group A sources all of which (with two exceptions) are located at $|b|<3^{\circ}$ - see lower panel of Fig. 4. The location of type A sources at low galactic latitude shows that they have almost certainly more massive progenitors. On the other hand, there are no long period counterparts to the (very likely less massive) high latitude sources. This means that they cannot reach stellar periods above $\sim 700$ days in the course of their evolution. The evolutionary status of E type sources located at low galactic latitudes is not so clear (see lower panel of Fig. 4). These could also be, similar to the high galactic latitude sources, low mass stars or they could be higher mass objects which form an evolutionary sequence with 

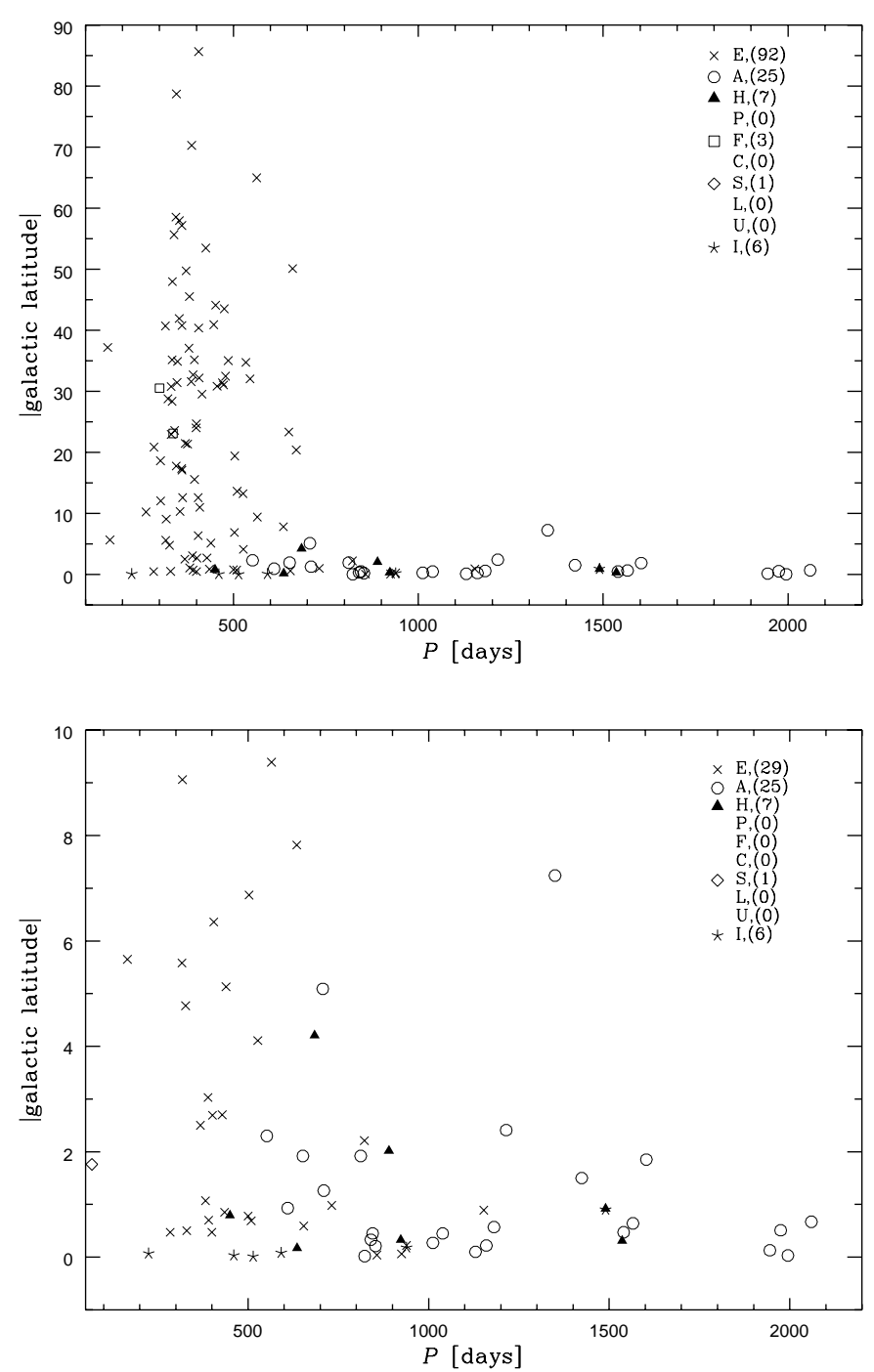

Fig. 4. The pulsational period-galactic latitude diagrams for $\mathrm{OH} / \mathrm{IR}$ stars from our sample: all sources - top diagram; source with $|b|<10^{\circ}$ - bottom diagram. Total number of sources plotted in each group is shown in parentheses

A type sources. It is worth noting that only low galactic latitude E type sources reach pulsational periods up to about 1000 days. It is possible that higher metallicity can play some role at least for some longer periods.

Note also that there is no clear period-colour relation for sources in group $\mathrm{H}$. In fact, the PPNe and $\mathrm{PNe}$ should be non-variables (Kwok 1987). This is also shown by their $\operatorname{VAR}(<40)$. Among the known PPNe and PNe in group H, only three: IRAS 07399-1435, IRAS 18135-1456 and IRAS 18276-1431 have known periods of 684, 450 and 890 days, respectively and it may indicate that these sources are early in the pre-planetary nebulae phase.

\subsection{Galactic distribution of $\mathrm{OH} / \mathrm{IR}$ stars}

The galactic distribution of all sources in the working sample is presented in Fig. 5, where symbols used for each group are the same as in Fig. 1. It is obvious that sources in group E cover a wide latitude range, whereas except for a few sources especially in group $F$ the remaining sources are located usually not more than 10 degrees from the galactic plane as the disk or bulge population. This distribution implies that high latitude sources in groups E and $\mathrm{F}$ are of different populations and must be relatively nearby objects whereas the remaining sources are statistically farther away. It is suggested that sources located in the galactic bulge or near the galactic plane are intrinsically brighter than those with high latitudes and have more massive progenitors (Volk \& Kwok 1987). We also noted that there is a sudden decline of the $\mathrm{OH} / \mathrm{IR}$ star population at longitudes of about +75 and -90 degree along with the galactic plane in Fig. 5 (these directions correspond to the local spiral arm), which is also seen in the CO distribution (Dame et al. 1987).

As has been already discussed (see Sect. 3.3), high galactic latitude group E sources apparently will never evolve into the region containing the reddest sources in groups $\mathrm{A}$ and $\mathrm{H}$, since the latter ones are all located at low latitudes near the galactic plane or in the galactic bulge. However, by plotting galactic coordinates for all objects from our working sample it becomes clearer that a significant fraction of group E objects have very low galactic latitudes. These sources also could have more massive progenitors (like type A objects) and will be able to evolve into objects with silicate absorption and then depart from the AGB as $\mathrm{H}$ sources (evolutionary sequence $\mathrm{A}-\mathrm{E}-\mathrm{H}$ for more massive stars) or they, independently of their galactic location, could be similar to lower mass high galactic latitude objects.

Some additional evidence for the presence of evolutionary and mass sequences among our working sample can be seen from the colour-latitude diagram in Fig. 6, which shows that there is a clear dependence of colour on galactic latitude. The group $\mathrm{E}$ objects have low $\log \left(F_{25} / F_{12}\right)$ colour and a large range of galactic latitudes, which demonstrates that our sample includes a significant fraction of nearby sources with Mira-like mass-loss rates, while type $\mathrm{A}$ source have larger $\log \left(F_{25} / F_{12}\right)$ colour and are more strongly confined to the galactic plane. This proves that the mass segregation is a function of infrared colour for OH/IR stars. As Likkel (1989) pointed out that the redder sources have a smaller scale height, and consequently have a larger initial mass (Miller \& Scalo 1979). In consequence, this implies that at least partly the colour sequence shown in Fig. 1 is a mass sequence (Likkel 1989), but the evolutionary sequence $(\mathrm{E}-\mathrm{A}-\mathrm{H})$ for more massive stars cannot be ruled out.

\subsection{Pumping efficiency of $\mathrm{OH} / \mathrm{IR}$ stars}

The correlated variations of $\mathrm{OH}$ and infrared flux densities (e.g. Harvey et al. 1974; Fillit et al. 1977; Werner et al. 1980) have shown that infrared radiation is the pumping mechanism for $\mathrm{OH}$ molecules produced through $\mathrm{H}_{2} \mathrm{O}$ photodissociation by interstellar ultraviolet radiation 


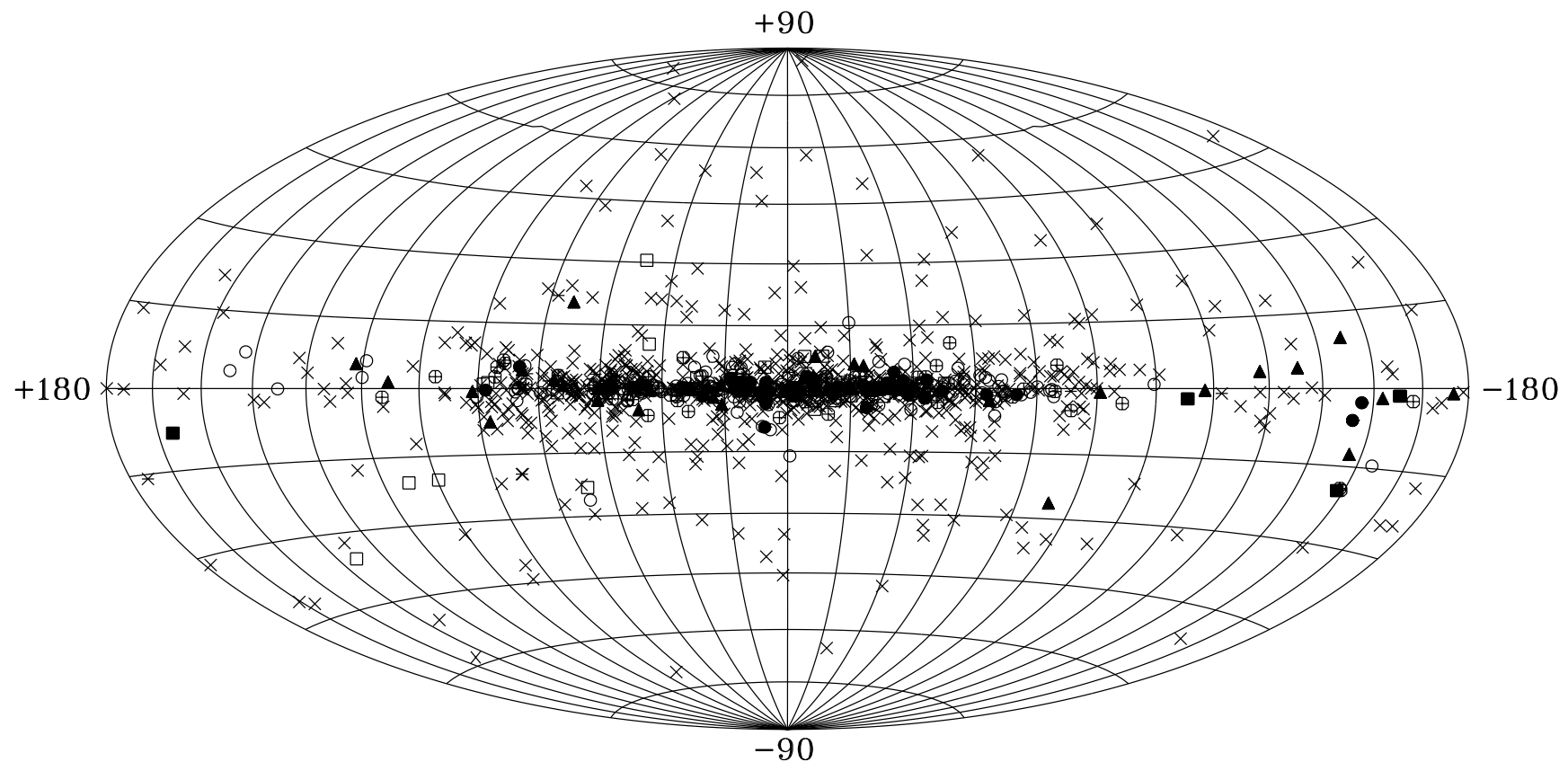

Fig. 5. The galactic distribution of $\mathrm{OH} / \mathrm{IR}$ stars in different LRS groups. The used symbols are the same as in Fig. 1

(Elitzur et al. 1976; Goldreich \& Scoville 1976). Although there were no direct measurements available before the Infrared Space Observatory (ISO) mission, it has been generally accepted that $1612 \mathrm{MHz} \mathrm{OH}$ masers are pumped by far infrared photons of dust thermal radiation mainly at about $35 \mu \mathrm{m}$ with possible contributions from photons at other wavelengths (e.g Elitzur et al. 1976; Bujarrabal et al. 1980; Thai-Q-Tung et al. 1998). Recently, the ISO Short Wavelength Spectrometer observations have indeed proved that there is an $\mathrm{OH}$ absorption line at $34.6 \mu \mathrm{m}$ (e.g. Justtanont et al. 1996; Sylvester et al. 1997; Barlow 1998). Other OH pumping lines has not been detected by ISO. Therefore, for the purpose of this paper, we consider the line around $35 \mu \mathrm{m}$ as the one which is the most important in creating the $\mathrm{OH}$ level inversion.

The pumping efficiency can be defined as $\epsilon=$ $n_{\mathrm{OH}} / n_{\mathrm{IR}}$, where $n_{\mathrm{OH}}$ is the number of $\mathrm{OH}$ photons emitted per second and $n_{\mathrm{IR}}$ is the number of the infrared photons absorbed per second in the $35 \mu \mathrm{m}$ rotational transitions. In the simplest case $\epsilon$ can be estimated by ratio of the $\mathrm{OH}$ peak flux density $\left(S_{\mathrm{OH}}\right)$ and infrared flux density $\left(F_{35}\right)$ at $35 \mu \mathrm{m}$ (Evans \& Beckwith 1977; Werner et al. 1980). Rottgering (1989) pointed out that the pumping efficiency should be calculated by means of the integrated fluxes. However, there is a strong correlation between the integrated flux and the corresponding peak flux density of $1612 \mathrm{MHz}$ maser emission (David et al. 1993). Furthermore, many observational works present only the peak flux densities (e.g. tLVHW, tL-H and most Nançay surveys), so the equation:

$\epsilon=S_{\mathrm{OH}} / F_{35}$

is used here to estimate the pumping efficiency for our sample of $\mathrm{OH} / \mathrm{IR}$ stars. We have assumed that $S_{\mathrm{OH}}$ is the average value of both the peak flux densities in the $1612 \mathrm{MHz}$ line. If only a single peak was detected, the second peak was assumed to have a peak flux density of zero. The infrared flux density at $35 \mu \mathrm{m}$ has been obtained (only when these fluxes had $Q=3$ or 2 ) by linear interpolation of the logarithms of the IRAS fluxes at 25 and $60 \mu \mathrm{m}$. The interpolated $F_{35}$ and $S_{\mathrm{OH}}$ flux densities in Jy are listed in Table 1 in Cols. 12 and 13, respectively. The lack of entry for $F_{35}$ in Table 1 means that PSC flux at 25 and/or $60 \mu \mathrm{m}$ had $Q=1$ ).

The histogram of pumping efficiency distribution defined by Eq. (1) for $\mathrm{OH} / \mathrm{IR}$ stars in groups $\mathrm{E}$ and $\mathrm{A}$ is presented in Fig. 7. In the last bin all values of $\epsilon$ larger than 0.45 have been accumulated. We do not consider here other groups since we suspect that they are not so uniform as groups $\mathrm{E}$ and $\mathrm{A}$. For example, in group $\mathrm{H}$ we have objects which belong to different classes: PPNe, PNe and HII regions (see Table 1 and Fig. 3), while group $\mathrm{P}$ and C sources which are probably surrounded by shells with carbon rich dust deserve special attention.

It is clear from Fig. 7 that pumping efficiencies for sources in group A are higher than for sources in group E. For most of the E group sources (about 98\%) we have $\epsilon$ smaller than 0.25 , while in the case of A sources this number is only about $86 \%$. It means that $\mathrm{OH}$ maser emission for most of E type sources can be explained in terms of infrared pumping model (where each maser photon requires at least about 4 infrared photons), while probably for some of the A group objects, either the predicted efficiencies are too small (see e.g. Thai-Q-Tung et al. 1998), or the $\epsilon$ 's estimated by Eq. (1) are too large. There is almost no time-coincidence between the IRAS flux densities and the $\mathrm{OH}$ data. It is easy to imagine that sources with infrared flux obtained at the minimum and $\mathrm{OH}$ maser 

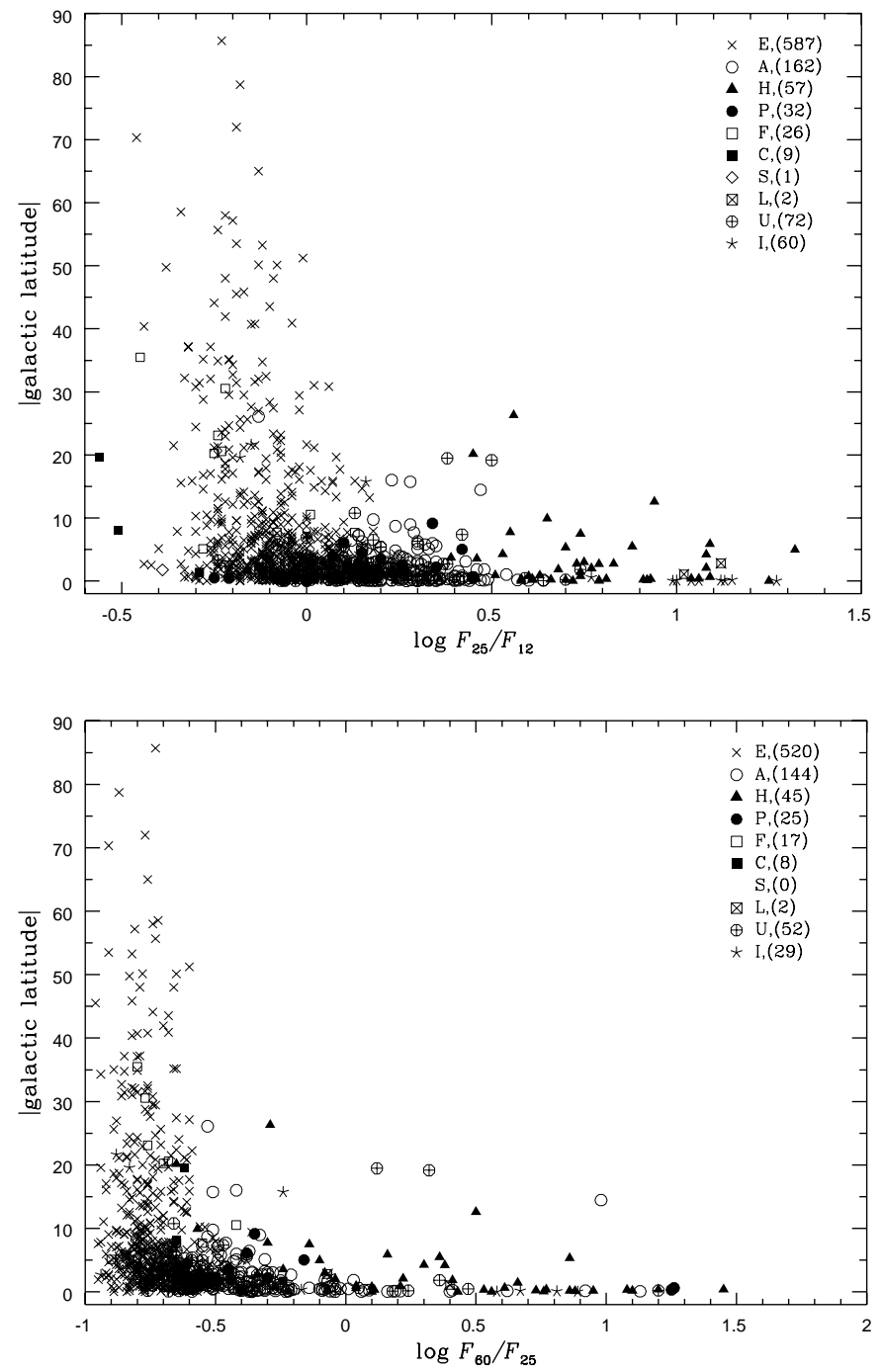

Fig. 6. The colour-galactic latitude diagrams of $\mathrm{OH} / \mathrm{IR}$ stars from our sample: galactic latitude versus $\log \left(F_{25} / F_{12}\right)$ (top diagram) and galactic latitude versus $\log \left(F_{60} / F_{25}\right)$ (bottom diagram). Numbers in parentheses show how many sources in each group have been used to construct the plot

measurements close to the maximum will show observational pumping efficiency much larger than the theoretical one.

In order to investigate the pumping efficiency for sources from group $\mathrm{A}$ and $\mathrm{E}$ in more detail the $\log S_{\mathrm{OH}}$ versus $\log F_{35}$ diagram is shown in Fig. 8 together with the linear regressions computed for sources of each group. We have found for sources in group $\mathrm{E}$ that the best fit relation is

$\log S_{\mathrm{OH}}=0.69( \pm 0.05) \log F_{35}-1.05( \pm 0.06)$

and for sources in group A the relation is

$\log S_{\mathrm{OH}}=1.13( \pm 0.09) \log F_{35}-1.23( \pm 0.15)$.

This confirms that the pumping efficiency is larger for sources from group A than for group $\mathrm{E}$ objects. Furthermore, from Eq. (3) (group A) we can see that $\mathrm{OH}$

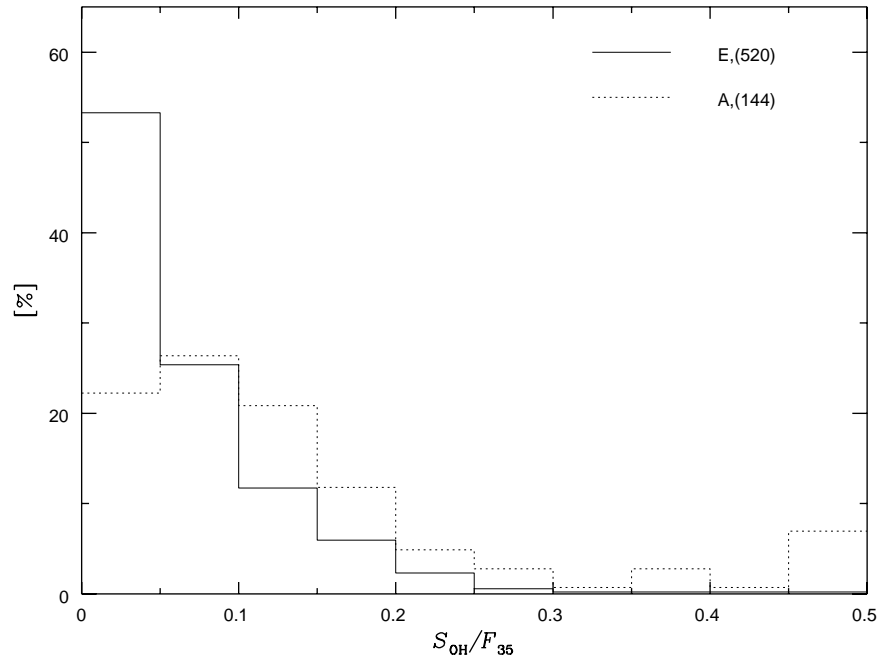

Fig. 7. The histogram of the pumping efficiency for $\mathrm{OH} / \mathrm{IR}$ stars in group E (solid line) and A (dotted line). Numbers in parentheses show how many sources in group $\mathrm{E}$ and $\mathrm{A}$ have been used to construct the plot

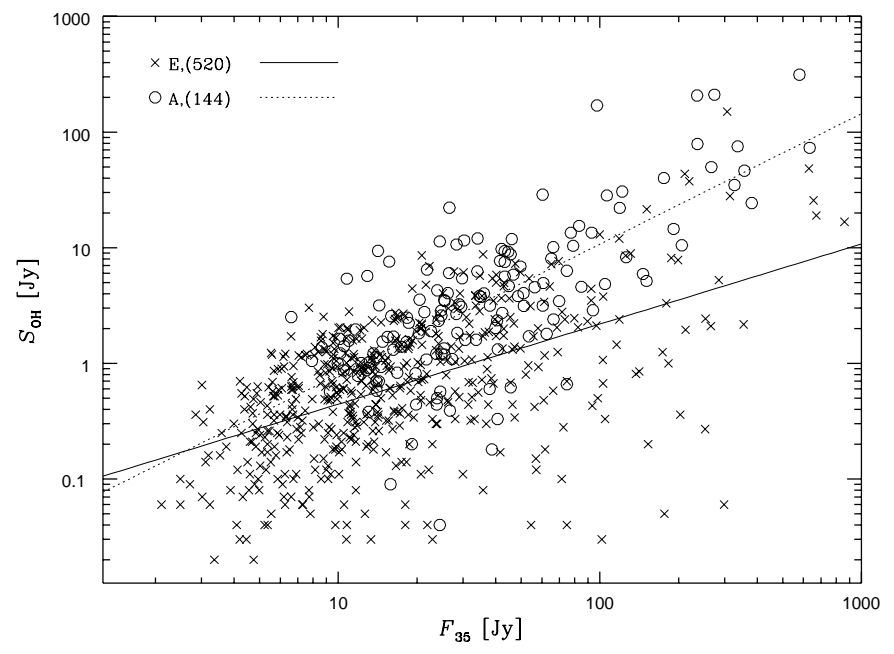

Fig. 8. The average value of two $\mathrm{OH}$ peak flux densities as a function of $F_{35}$ for sources in group $\mathrm{E}$ and $\mathrm{A}$. The linear regression is shown by means of solid line for sources from group E and dotted line from those in group A. Total number of sources plotted in each group is shown in parentheses

maser flux is almost proportional to the infrared flux at $35 \mu \mathrm{m}$, while this is not the case for group E sources (Eq. (2)). The large scatter of data seen in Fig. 8 may be partly due to stellar and, related to it, $\mathrm{OH}$ maser variations.

We note that observational estimates of the pumping efficiencies are strongly biased towards higher values by the detection levels in the $\mathrm{OH}$ surveys, i.e. it is easier to detect strong $\mathrm{OH}$ sources with higher efficiencies. However, in spite of the fact that the sample selected by us is rather non-uniform in terms of the $\mathrm{OH}$ detection thresholds, we are convinced that the size of our data base allows us to determine average pumping efficiencies for different groups and to attribute statistical significance to these values. 


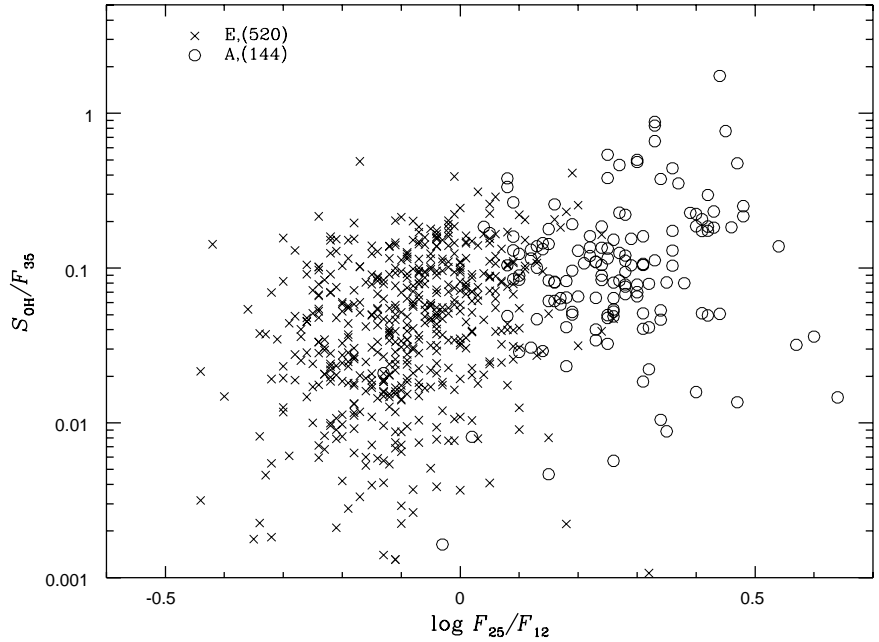

Fig. 9. Pumping efficiency as a function of $\log F_{25} / F_{12}$ colour for sources in group $\mathrm{E}$ and $\mathrm{A}$. Total number of sources plotted in each group is shown in parentheses

The average value of pumping efficiency for A sources is $0.10 \pm 0.03$ and it is $0.03 \pm 0.02$ for E objects. Here, errors have been estimated using Eqs. (3) and (2) for sources in group $\mathrm{A}$ and $\mathrm{E}$, respectively. With the above mentioned bias, the true averaged efficiencies for the A and E group will be probably somewhat lower than values calculated from the assembled observations. Nevertheless, the obtained result is likely to be statistically significant and shows that sources in group A, on average, are more efficient (about 3 times) than sources in group $\mathrm{E}$ in pumping the $1612 \mathrm{MHz}$ maser emission. This probably reflects the larger thickness and higher optical depth for circumstellar dust shell around group A sources. We note that the results we obtained from the present sample (520 sources from group E and 144 from group A) are in agreement with previously published results using smaller samples. For example, Dickinson (1987), using data for $163 \mathrm{OH} / \mathrm{IR}$ stars, found an average pumping efficiency of 0.12 for sources that have fluxes peaking in $12-80 \mu \mathrm{m}$ (sources with prominent dust shell), and of $0.05-0.06$ for sources that have fluxes falling from 12 to beyond $80 \mu \mathrm{m}$ (small contribution from the circumstellar dust). Silva et al. (1993), using data for $10 \mathrm{OH} / \mathrm{IR}$ stars, found an average pumping efficiency of 0.11 by using the linear interpolation and 0.13 by using the blackbody fitting, respectively. However after inspection of their sample, we found that $5 \mathrm{OH} / \mathrm{IR}$ stars have the LRS spectra classifying them as objects of group A. Recent theoretical results of Thai-Q-Tung et al. (1998) predict pumping efficiencies from about 0.05 up to 0.17 for the saturated masers, which are in agreement with the averaged values found in this work.

Finally, in Fig. 9 we present the pumping efficiency versus colour diagram for sources in groups $\mathrm{A}$ and $\mathrm{E}$. It is clear that the pumping efficiency increases with the infrared colour. It means that the pumping efficiency becomes higher when the optical thickness of the envelope, or the mass loss rate, reach larger values, i.e. for stars with

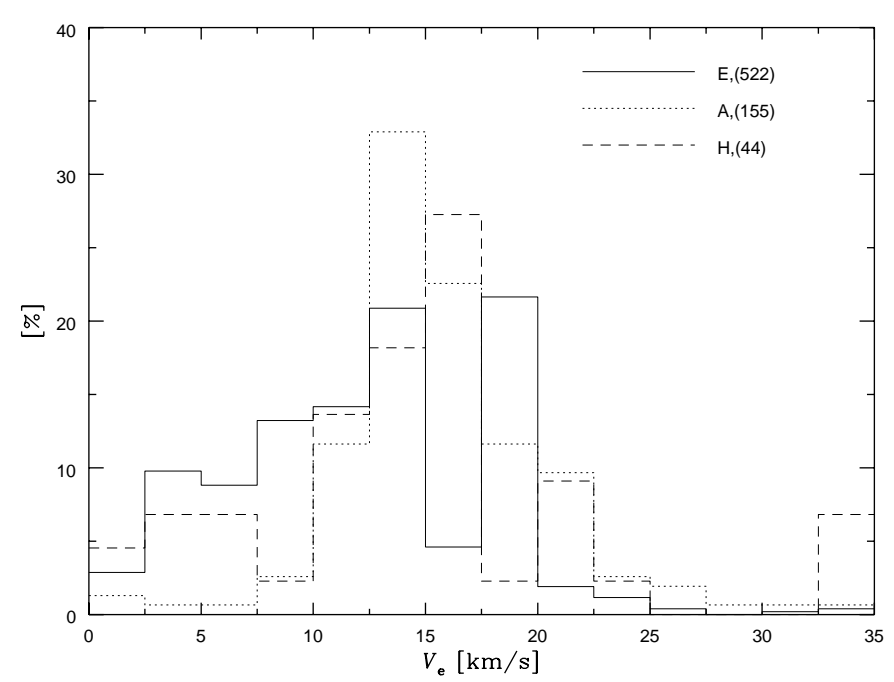

Fig. 10. The histogram of the envelope expansion velocities for $\mathrm{OH} / \mathrm{IR}$ stars in groups $\mathrm{E}$ (solid), A (dotted) and $\mathrm{H}$ (dashed line). Numbers in parentheses show how many sources in each group have been used to construct the histogram

silicate absorption feature at $9.7 \mu \mathrm{m}$ (A). Elitzur (1981) has mentioned that when mass loss is high enough the far infrared transitions become optically thick and the maser flux is proportional to the far infrared flux, whereas in the optically thin case the pump rate is proportional to not only the infrared flux but also to the number of absorbing $\mathrm{OH}$ molecules. These theoretical predictions are supported by our empirical relationships given in Eqs. (3) and (2), respectively.

\subsection{Envelope expansion velocity of $O H / I R$ stars}

It is generally accepted that the presence or absence of an $\mathrm{OH}$ maser depends on the local density of the envelope at the radius of interest, which depends on both the mass loss rate and the shell expansion velocity (Lewis 1989). Assuming the classical picture of a spherical, thin and uniformly expanding dust shell, the envelope expansion velocity $V_{\mathrm{e}}$ can be estimated as a half of the velocity difference between the two $1612 \mathrm{MHz}$ peaks. The histogram of the shell expansion velocities for $\mathrm{OH} / \mathrm{IR}$ stars in groups $\mathrm{E}, \mathrm{A}$ and $\mathrm{H}$ is shown in Fig. 10, where in the last bin we have accumulated all velocities larger than $32.5 \mathrm{~km} \mathrm{~s}^{-1}$. From the inspection of this histogram we can see that for most of the sources $V_{\mathrm{e}}$ lies between 10 and $20 \mathrm{~km} \mathrm{~s}^{-1}$ (58.5, 77.6 and $61.4 \%$ for sources from group E, A and $\mathrm{H}$, respectively) as commonly found in the previous work (e.g. Herman 1983; Le Squeren et al. 1992). Also it is found that, on the average, $V_{\mathrm{e}}$ for sources in group $\mathrm{A}$ is larger than that in group $\mathrm{E}(34.6,5.3$ and $20.5 \%$ of the stars have $V_{\mathrm{e}}$ smaller than $10 \mathrm{~km} \mathrm{~s}^{-1}$, for sources from group E, $\mathrm{A}$ and $\mathrm{H}$, respectively). Note also that it is mainly sources from groups $\mathrm{A}$ and $\mathrm{H}$ which have shell velocities larger than $20 \mathrm{~km} \mathrm{~s}^{-1}$. 


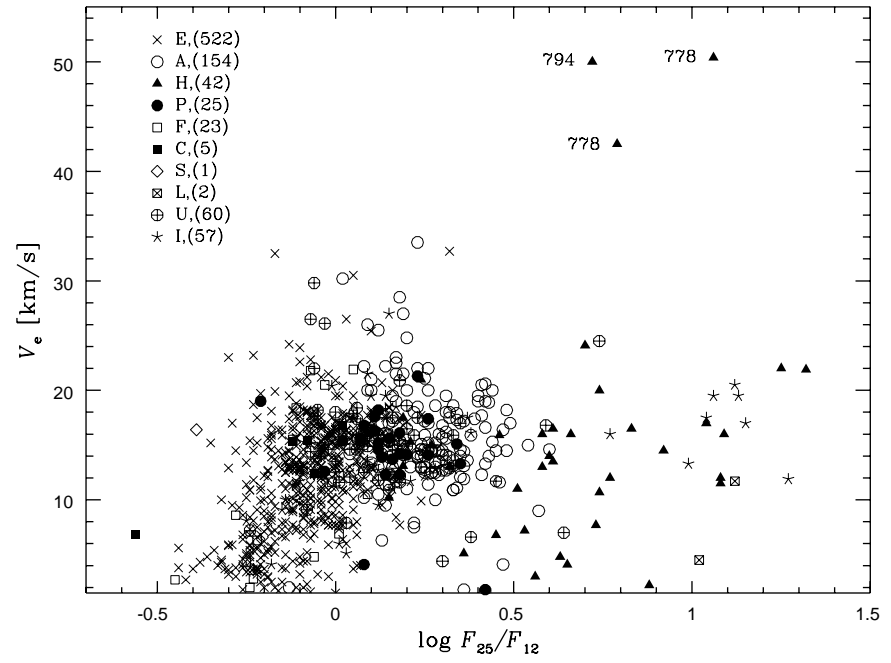

Fig. 11. The $V_{\mathrm{e}}$ versus $\log F_{25} / F_{12}$ diagram for all $\mathrm{OH} / \mathrm{IR}$ stars. Some extreme expansion velocity sources are marked by their numbers from Table1. Total number of sources plotted in each group is shown in parentheses

In order to quantitatively test this idea, we have plotted in Fig. 11 the $\log F_{25} / F_{12}$ colour- $V_{\text {e }}$ diagram for all $\mathrm{OH} / \mathrm{IR}$ stars. Although the data suffer from considerable scatter, it is clear that the expansion velocity increases with $\log F_{25} / F_{12}$ for bluer colour objects (groups $\mathrm{E}$ and $\mathrm{F}$ ), whereas the expansion velocity is weakly dependent (if at all) on the infrared colour for the redder sources (groups A, $\mathrm{P}$ and $\mathrm{H}$ ). This result is in agreement with previous ones (e.g. Lewis 1989; Le Squeren et al. 1992; David et al. 1993; Chengalur et al. 1993). Note also that 3 sources in group $\mathrm{H}$ (they are identified by their numbers from Table 1) have extremely high expansion velocities. Zijlstra et al. (1989) suggested that such objects may be post-AGB stars which will soon evolve into PNe. In fact, one of them IRAS 17347-3139 (794) is classified as PPN object.

Similar patterns can also be seen in the period- $V_{\mathrm{e}}$ diagram in Fig. 12. The expansion velocity gradually increases with the period for sources in groups E, but at period about 600 days it keeps a constant value of about $15 \mathrm{kms}^{-1}$ with a dispersion of $\pm 5 \mathrm{~km} \mathrm{~s}^{-1}$ for sources in groups A and H. According to Baud et al. (1981) the expansion velocity is a measure of the main sequence mass so that, on the average, the redder the envelope, the larger the initial mass. Thus also from the $V_{\mathrm{e}}$ behaviour, sources in groups $\mathrm{E}$ would have a wider range of initial masses whereas sources in groups $\mathrm{A}, \mathrm{P}$ and $\mathrm{H}$ would all be rather massive stars. Again this means that only massive $\mathrm{OH} / \mathrm{IR}$ stars can evolve to very red colour with very high mass loss rate as proposed by Volk \& Kwok (1987) and Likkel (1989). The pattern of behaviour of the expansion velocities presented in Figs. 11 and 12 can be understood on the basis of the efficiency of photon momentum transfer to the gas shell: when the mass loss is small any increase causes a larger dust-gas coupling and so the momentum transfer increases, while once a critical value of mass loss

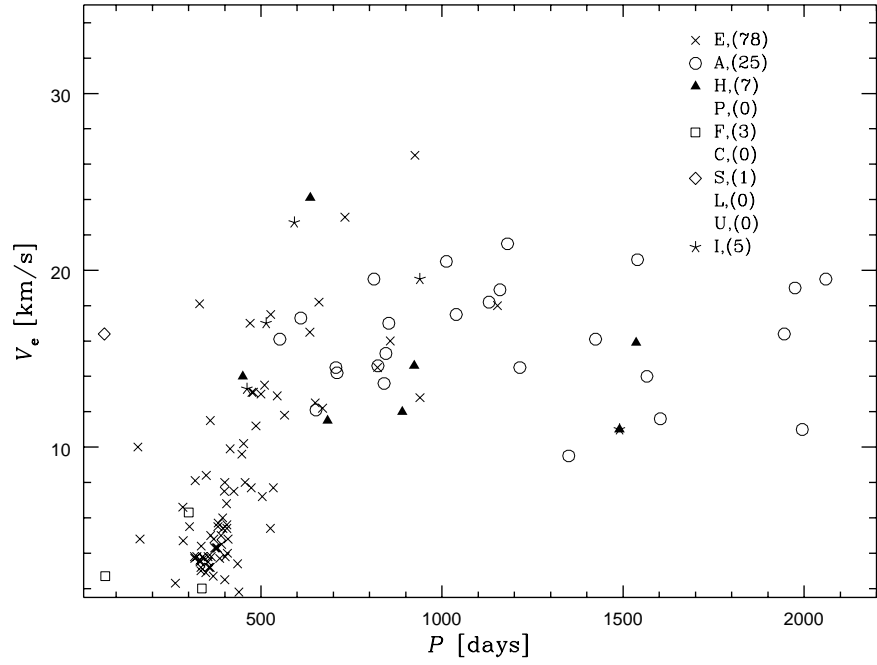

Fig. 12. The $V_{\mathrm{e}}$ versus $P$ diagram for all $\mathrm{OH} / \mathrm{IR}$ stars. Total number of sources plotted in each group is shown in parentheses

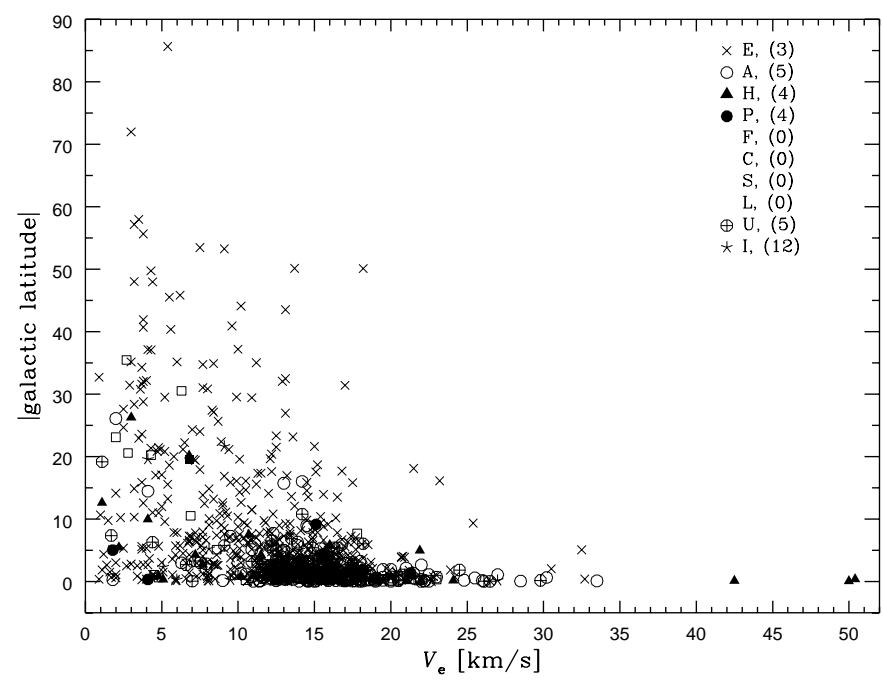

Fig. 13. The galactic latitude versus $V_{\mathrm{e}}$ diagram for all $\mathrm{OH} / \mathrm{IR}$ stars. Total number of sources plotted in each group is shown in parentheses

is reached the further increase in the momentum transfer and thus gas acceleration is not possible (see e.g. Kwok 1975; Chengalur et al. 1993; Steffen et al. 1997, 1998).

From the galactic latitude versus $V_{\mathrm{e}}$ diagram shown for all OH/IR stars in Fig. 13, it is seen that sources with high expansion velocity (over $15 \mathrm{~km} \mathrm{~s}^{-1}$ ) are located mainly along the galactic plane, whereas in high latitude regions $\left(|b|>10^{\circ}\right)$ almost there are no sources with the expansion velocity larger than about $20 \mathrm{~km} \mathrm{~s}^{-1}$. This again can be interpreted as the signature of the shell velocity dependence on the mass of the star: the former sources are thought to be more massive $\mathrm{OH} / \mathrm{IR}$ stars and many of the later are sources with low initial mass.

Another interesting result is presented in Fig. 14, where sources with $\left(|b|<10^{\circ}\right)$ and $\left(|l|<15^{\circ}\right)$ are taken as galactic bulge objects (subsample includes 99 and 


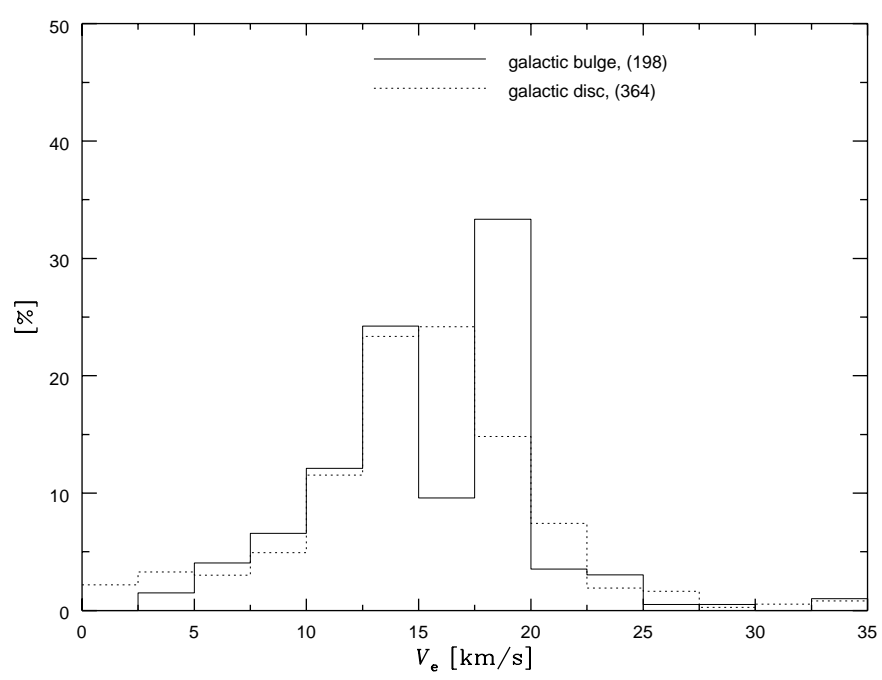

Fig. 14. The histogram of the shell expansion velocity for $\mathrm{OH} / \mathrm{IR}$ stars in the galactic bulge (solid) and the galactic disc (dotted). Numbers in parentheses show how many sources have been used to construct the histogram

44 objects of group E and A, respectively), and sources with $\left(|b|<3^{\circ}\right)$ and $\left(|l|>15^{\circ}\right)$ (here 182 objects belong to group E and 96 to group A) are taken as the galactic disk objects. It is seen that, on the average, both group of the sources have essentially similar distributions of the $V_{\mathrm{e}}$. Metallicity in the galactic bulge is thought to be larger than that in the disk so the expansion velocity should be larger, but on the other hand the density of the interstellar matter is higher as well so as a result both groups of objects have similar $V_{\mathrm{e}}$ values.

\subsection{Galactic longitude-radial velocity distribution}

The Local Standard of Rest (LSR) stellar velocities of $\mathrm{OH} / \mathrm{IR}$ stars are derived as the average velocity of the two peaks of $\mathrm{OH} 1612 \mathrm{MHz}$ maser emission. In the case when only one peak is observed the peak velocity is treated as the stellar radial velocity. The distribution of radial velocity with galactic longitude can be used to study galactic kinematics by considering how closely $\mathrm{OH} / \mathrm{IR}$ stars follow the expected galactic rotation curve. However at higher latitude the sample (mainly in group E) is dominated by nearby stars with low initial mass, as previously discussed. The following discussion is restricted to $\mathrm{OH} / \mathrm{IR}$ stars with galactic latitudes $|b|<10^{\circ}$. This includes almost all galactic bulge and disk population objects but excludes most nearby objects. The galactic longitude-radial velocity distribution for all $\mathrm{OH} / \mathrm{IR}$ stars with $|b|<10^{\circ}$ is illustrated in Fig. 15, in which we can see the presence of the counter-rotating $\mathrm{OH} / \mathrm{IR}$ stars (especially in the bulge region) which point to orbits that are not simply circular. The galactic rotation is best represented by the $\mathrm{CO}$ emission, and the map of the radial velocity distribution of $\mathrm{CO}$ has been given by Dame et al. (1987). It is found that in general the shape of the distribution of most $\mathrm{OH} / \mathrm{IR}$ stars

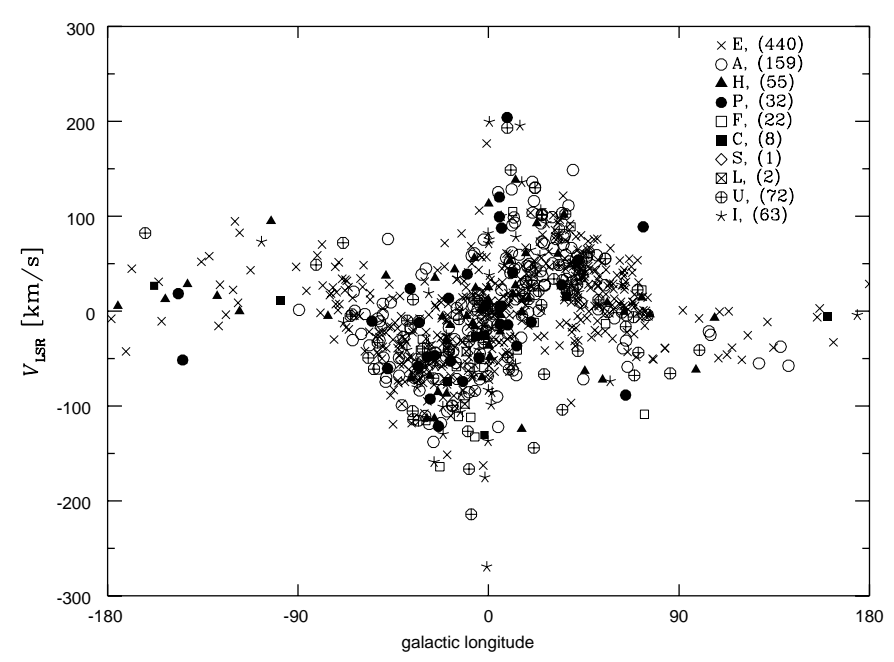

Fig. 15. The galactic longitude distribution of the stellar LSR radial velocity for all $\mathrm{OH} / \mathrm{IR}$ stars with $|b|<10^{\circ}$. Total number of sources plotted in each group is shown in parentheses

is very similar to that of CO in Dame's Fig. 6 which follows the galactic rotation curve. Further inspection shows that $\mathrm{OH} / \mathrm{IR}$ stars in group $\mathrm{A}$ are clustered in the molecular ring which exhibits strong $\mathrm{CO}$ emission, and which is seen as the diagonal line within $30^{\circ}$ of the galactic center. In addition, outside the solar circle only 3 sources in group A are seen. Finally, the sources in the galactic bulge follow the rotation curve while the sources at the galactic center indicate a mass concentration (Dejonghe 1993).

\section{Summary and conclusions}

In this paper we assembled $1065 \mathrm{OH} / \mathrm{IR}$ sources (mainly AGB and post-AGB stars) with IRAS LRS spectra to investigate the properties of $\mathrm{OH} / \mathrm{IR}$ stars by combining their characteristics in the infrared and in $\mathrm{OH} 1612 \mathrm{MHz}$ maser emission. Among these sources 1024 have good IRAS associations with a difference between the IRAS and $\mathrm{OH}$ maser positions of smaller than $1^{\prime}$. Our sample has been created by cross-correlation of $\mathrm{OH}$ satellite line maser data (from predominantly four large $\mathrm{OH}$ surveys: $\mathrm{tL}-\mathrm{H}, \mathrm{AO}$, tLVHW and ATCA) with the recent catalogue of the IRAS LRS spectra (Kwok et al. 1997) which have applied new classification scheme of LRS spectra developed by Volk \& Cohen (1989). About 90\% of the analyzed sample come from these surveys. Data for an additional $105 \mathrm{OH} / \mathrm{IR}$ sources have been taken from more than 20 papers (see Sect. 2 for details). The assembled data base is rather non-uniform: the different surveys have different detection thresholds and vary from inaccurate radio positions for the single dish surveys (e.g. included in the pre-IRAS blind observations catalogue - tLVHW) to highly accurate positions for the interferometric surveys. However, full analysis of the completeness and uniformity of our sample is outside of scope of this paper. On the other hand, our sample is sufficiently large that most (very likely all) our conclusions of a statistical nature are probably correct. 
The majority of $\mathrm{OH} / \mathrm{IR}$ stars at the AGB phase of evolution have silicate features either in emission or in absorption. However, we have also a significant number of sources with $\mathrm{OH}$ maser emission for which classification of their LRS spectra suggest that their circumstellar envelopes are carbon rich. Recently, ISO discovered that simultaneous appearance of oxygen- and carbon-rich material around stars is quite common in different stages of their life. Therefore, our finding is perhaps not so surprising but, nevertheless, these sources deserve special attention and work on understanding their nature is in progress. In contrast to the common assumption that $\mathrm{OH} / \mathrm{IR}$ stars represent extremely evolved AGB stars, we found that sources with silicate emission (group E) exceed in number the sources with silicate absorption (group A). When we examine data from separate $\mathrm{OH}$ surveys, we found that the relative number between $\mathrm{E}$ and $\mathrm{A}$ group sources increases significantly when sensitivity of survey is larger, independent of the source selection criteria: AO (IRAS selected sample) and ATCA (blind observations of galactic bulge and galactic disc). Therefore, we are convinced that larger number of $\mathrm{OH}$ maser detection in E group sources is real and represent a true phenomenon: probably it is related to larger number of the IRAS sources with silicate emission. But it also shows that physical conditions in shells around group $\mathrm{E}$ objects are sufficient to pump the $\mathrm{OH}$ masers.

Analysis of various diagrams relating different physical parameters of $\mathrm{OH} / \mathrm{IR}$ sources from our sample allowed us to demonstrate that there is a clear mass sequence, especially, among type $\mathrm{E}$ and $\mathrm{A}$ sources. Most of the E sources have a smaller initial mass than A group objects and they will never evolve to develop circumstellar shells thick enough to show the silicate absorption feature. The mass sequence is hidden in data presented on the IRAS colourcolour diagram as well as in the period-colour diagram but is clearly seen in diagrams which relate different physical parameters with galactic distribution (galactic latitude). Since the IRAS colour-colour diagram has been frequently used for discussion of properties and evolutionary status of AGB stars it is obvious why an evolutionary sequence (the silicate feature changes from emission to absorption as mass loss of star increases) was the most "widely accepted". We note that earlier works have suggested that a mass sequence is probably mixed with an evolutionary one. On the other hand, our results show that mass sequence is more prominent in many of the observed properties but also suggest the existence of an evolutionary sequence $(\mathrm{E}-\mathrm{A}-\mathrm{H})$ for more massive stars.

Let us summarize differences between the most numerous objects in our sample (E, A and $\mathrm{H}-$ about $80 \%$ of all sources - if not explicitly stated the discussed results refer to these groups). While most of the following results were already known from previous studies, the size of our sample allows to treat our results as more conclusive. The most interesting result is a remarkable difference in galactic location between $\mathrm{OH} / \mathrm{IR}$ sources with the silicate emission and absorption feature. We found only a few sources of type A at galactic latitude $>10^{\circ}$. Consequences of this finding were already discussed above. Note that only $\mathrm{OH} / \mathrm{IR}$ sources with $|b|<10^{\circ}$ follow the galactic rotation curve and that $\mathrm{OH} / \mathrm{IR}$ stars in group A are clustered along with molecular ring which is seen as the diagonal line within $30^{\circ}$ of the galactic center. On the IRAS colour-colour diagram OH/IR stars follow the well known behaviour established for oxygen-rich AGB and post-AGB stars. Sources in groups E and A have very high probabilities of variability, while sources in group $\mathrm{H}$ have much lower ones. This suggests that $\mathrm{OH} / \mathrm{IR}$ stars in groups $\mathrm{E}$ and $\mathrm{A}$ are undergoing mass loss whereas most sources in group H may have stopped process of intense mass loss and are evolving directly to the planetary nebula region. Stellar pulsation periods for group E objects increases with the IRAS colours while for group A sources the correlation between period and colour essentially breaks down and at the same colour we can find periods of widely different values. The sources in group E have periods of about several hundred days (with exception of low galactic latitude sources which could reach periods as high as 1000 or more days) and sources in group A have periods from about 500 to over 2000 days. Shell expansion velocities for group $\mathrm{A}$ and $\mathrm{H}$ are rather uniform (concentrated around $15-25 \mathrm{~km} \mathrm{~s}^{-1}$ ) and statistically larger than those for group $\mathrm{E}$ objects. In the case of silicate emission sources the expansion velocity increases with $\log F_{25} / F_{12}$. Pumping efficiencies for group $\mathrm{E}$ and $\mathrm{A}$ sources also increase with colour but they are statistically higher for sources in group A. This probably reflects the larger thickness and higher optical depth for circumstellar dust shell around group A sources.

Acknowledgements. We are very indebted to the referee Dr. J. Chapman whose very careful and inspiring comments allowed us to improve this manuscript. We thank Dr. B. M. Lewis for sending us his unpublished catalogue. This work is in part supported by grant from the Natural Science and Engineering Research Council of Canada, the National Natural Science Foundation of China and the grant 2.P03D.002.13 to RS from the Polish State Committee for Scientific Research.

\section{References}

Barlow, M. J. 1998, Ap\&SS, 255, 315

Baud, B., \& Habing, H. J. 1983, A\&A, 127, 73

Baud, B., Habing, H. J., Matthews, H. E., et al. 1979a, A\&AS, 35, 179

Baud, B., Habing, H. J., Matthews, H. E., et al. 1979b, A\&AS, 36, 193

Baud, B., Habing, H. J., Matthews, H. E., et al. 1981, A\&A, 95, 156

Bedijn, P. J. 1987, A\&A, 186, 136

Blommaert, J. A. D. L., van Langevelde, H. J., \& Michids, W. F. P. 1994, A\&A, 287, 479 (BLW94)

Bowers, P. F. 1978a, A\&AS, 31, 127

Bowers, P. F. 1978b, A\&A, 64, 307

Braz, M. A., Lépine, J. R. D., Sivagnanam, P., et al. 1990, A\&A, 236, 479 (BLS90)

Bujarrabal, V., Guibert, J., Nguyen-Q-Rieu, et al. 1980, A\&A, 84,311 
Caswell, J. L., \& Haynes, R. F. 1975, MNRAS, 173, 649

Caswell, J. L., Haynes, R. F., Goss, W. M., et al. 1981, Aust. J. Phys., 34, 333

Chan, S. J., \& Kwok, S. 1988, ApJ, 334, 362

Chan, S. J., \& Kwok, S. 1990, A\&A, 237, 354

Chengalur, J. N., Lewis, B. M., Eder, J., et al. 1993, ApJS, 89, $189(\mathrm{AO})$

Dame, T. M., Ungerechts, H., Cohen, R. S., et al. 1987, ApJ, 322,706

David, P., Le Squeren, A. M., \& Sivagnanam, P. 1993, A\&A, 277, 453 (DSS93)

Dejonghe, H. 1993, in Galactic Bulge, ed. H. Dejonghe, \& H. J. Habing (Kluwer Academic Pub.), 57

Dickinson, D. F. 1987, ApJ, 313, 408

Dickinson, D. F., \& Chaisson, E. J. 1973, ApJ, 181, L135 (DC73)

Eder, J., Lewis, B. M., \& Terzian, Y. 1988, ApJS, 66, 183 (AO)

Engels, D. 1979, A\&AS, 36, 337 (EN79)

Elitzur, M., Goldreich, P., \& Scoville, N. 1976, ApJ, 205, 384

Elitzur, M. 1981, in Physical Processes in Red Gaints, ed. I. Iben, A. Renzini, \& D. Dordrecht (Reidel Publishing Co.), 363

Elitzur, M. 1992, Astronomical Masers (Kluwer Academic Publishers)

Evans, N. J., \& Beckwith, S. 1977, ApJ, 217, 729

Fillit, R., Proust, D., \& Lepine, J .R. D. 1977, A\&A, 58, 281

Frail, D. A., \& Beasley, A. J. 1994, A\&A, 290, 796 (FB94)

Galt, J. A., Kwok, S., \& Frankow, J. 1989, AJ, 98, 2182 (GKF89)

Gaylard, M. J., \& Whitelock, P. A. 1988, MNRAS, 235, 123 (GW88)

Gaylard, M. J., West, M. E., Whitelock, P. A., et al. 1989, MNRAS, 236, 247 (GWW89)

Goldreich, P., \& Scoville, N. 1976, ApJ, 205, 144

Habing, H. J., Olnon, F. J., Winnberg, A., et al. 1983, A\&A, 128,230

Harvey, P. M., Bechis, K. P., Wilson, W. J., et al. 1974, ApJS, 27,331

Herman, J. 1983, Thesis, University of Leiden

Herman, J., \& Habing, H. J. 1985, Phys. Rep., 124, 255

HST Guide Star Catalogue, 1989, Baltimore: STScI, Astronomical Data Center CD-ROM Selected Astronomical Catalogue, 1 (HST GSC)

IRAS Point Source Catalogue, Version 2, 1988, Joint IRAS Science Working Group, Washington, DC (PSC)

Ivison, R. J., Seaquist, E. R., \& Hall, P. J. 1994, MNRAS, 269, 218 (ISH94)

Johansson, L. E. B., Andersson, C., Goss, W. M., et al. 1977a, A\&AS, 28199

Johansson, L. E. B., Andersson, C., Goss, W. M., et al. 1977b, A\&A, 54, 323

Justtanont, K., de Jong, Th., Helmich, F. P., et al. 1996, A\&A, 315, L217

Kholopov, P. N., Samus, N. N., Frolov, M. S., et al. 19851988, General Catalogue of Variable Stars 4th ed. (Moscow, Nauka)

Kleinmann, S. G., Gillet, F. C., \& Joyce, R. R. 1981, ARA\&A, 19, 411

Kwok, S. 1975, ApJ, 198, 583

Kwok, S. 1976, J. Roy. Astr. Soc. Canada, 70, 49

Kwok, S. 1987, Phys. Rep., 156, 113

Kwok, S. 1990, MNRAS, 244, 179

Kwok, S., Volk, K., \& Bidelman, W. P. 1997, ApJS, 112, 557
Le Squeren, A. M., Sivagnanam, P., Dennefeld, M., et al. 1992, A\&A, 254, 133 (SSD92)

Lewis, B. M. 1989, ApJ, 338, 234

Lewis, B. M., Eder, J., \& Terzian, Y. 1990, ApJ, 362, 634 (AO)

Lewis, B. M. 1992a, ApJ, 396, 251 (AO)

Lewis, B. M. 1992b, private communication to S. Kwok, Nov. 22 (Lewis Catalogue)

Lewis, B. M. 1994, ApJS, 93, 549 (AO)

Likkel, L. 1989, ApJ, 344, 350 (L89)

Lindqvist, M., Winnberg, A., Habing, H. J., et al. 1992, A\&AS, 92, 43 (LWH92)

Miller, G. E., \& Scalo, J. M. 1979, ApJS, 41, 513

Olnon, F. M., Walterbos, R. A., Habing, H. J., et al. 1981, ApJ, 245, L103

Olnon, F. M., Baud, B., Habing, H. J., et al. 1984, ApJ, 278, L41

Omont, A., Loup, C., Forveille, T., et al. 1993, A\&A, 267, 515

Rottgerring, H. J. A. 1989, A\&A, 222, 125

Sevenster, M. N., Chapman, J. M., Habing, H. J., et al. 1997a, A\&AS, 122, 79 (ATCA)

Sevenster, M. N., Chapman, J. M., Habing, H. J., et al. 1997b, A\&AS, 124, 509 (ATCA)

Silva, A. M., Azcarete, I. N., Poppel, W. G. L., et al. 1993, A\&A, 275, 501 (SAP93)

Sivagnanam, P., Le Squeren, A. M., \& Foy, F. 1988, A\&A, 206, 285 (SSF88)

Sivagnanam, P., Le Squeren, A. M., Foy, F., et al. 1989, A\&A, 211,341

Sivagnanam, P., Braz, M. A., Le Squeren, A. M., et al. 1990, A\&A, 233, 112 (SBS90)

Sivagnanam, P., \& Le Squeren, A. M. 1990, From Miras to Planetary Nebulae: Which Path for Stellar Evolution? ed. M. O. Mennessier, \& A. Omont, Proceedings of the International Colloquium, 225

Sjouwerman, L. O., van Langevelde, H. J., Winnberg, A., et al. 1998, A\&AS, 128, 35 (SLW98)

Steffen, M., Szczerba, R., Men'schikov, A., et al. 1997, A\&AS, 126,39

Steffen, M., Szczerba, R., \& Schönberner, D. 1998, A\&A, 337, 149

Stephenson, C. B. 1989, A general catalogue of cool galactic carbon stars, Second edition (Pub. Warner \& Swasey Obs. 3), part 2, 55 (CCGCS)

Sun, J., \& Kwok, S. 1987, A\&A, 185, 285

Sylvester, R. J., Barlow, M. J., Liu, X. W., et al. 1997, MNRAS, 291, L42

te Lintel Hekkert, P., Habing, H. J., Caswell, J. L., et al. 1988, A\&A, 202, L19 (LHC88)

te Lintel Hekkert, P., Versteege-Hensel, H. A., Habing, H. J., et al. 1989, A\&AS, 78, 399 (tLVHW)

te Lintel Hekkert, P. 1990, Ph.D. Thesis, University of Leiden (tL-H)

te Lintel Hekkert, P. 1991, A\&A, 248, 209 (tLH91)

te Lintel Hekkert, P., Caswell, J. L., Habing, H. J., et al. 1991, A\&AS, 90, 327 (tL-H)

te Lintel Hekkert, P., \& Chapman, J. M. 1996, A\&AS, 119, 459 (LC96)

Thai-Q-Tung, Dinh-V-Trung, Nguyen-Q-Rieu, et al. 1998, A\&A, 331, 317

van der Veen, W. E. C. J., Habing, H. J., \& Geballe, T. 1987, in Planetary and Proto-Planetary Nebulae: from IRAS to ISO, Proceedings of the Frascati Workshop, ed. A. P. Martinez (Dordrecht, D. Reidel Publishing Co.), 69 
van der Veen, W. E. C. J., \& Habing, H. J. 1988, A\&A, 194, 125

van Langevelde, H. J., Brown, A. G. A., Lindqvist, M., et al. 1992, A\&A, 261, L17 (LBL92)

Volk, K., \& Kwok, S. 1987, ApJ, 315, 654

Volk, K., \& Kwok, S. 1988, ApJ, 331, 435

Volk, K., \& Cohen, M. 1989, AJ, 98, 931

Volk, K., \& Cohen, M. 1990, AJ, 100, 485

Volk, K., Kwok, S., \& Langill, P. P. 1992, ApJ, 391, 285

Weaver, H., Williams, D. R. W., Dieter, N. H., et al. 1965, Nature, 208, 29

Werner, M. W., Beckwith, S., Gatley, I., et al. 1980, ApJ, 239, 540
Whitelock, P., Menzies, J., Feast, M., et al. 1994, MNRAS, 267, 711 (WMF94)

Wilson, W. J., \& Barrett, A. H. 1968, Science, 161, 728

Wilson, W. J., \& Barrett, A. H. 1972, A\&A, 17, 185

Winnberg, A., Nguyen-Q-Rieu, Johansson, L. E. B., et al. 1975, A\&A, 38, 145

Wood, P. R., Habing, H. J., \& McGregor, P. J. 1998, A\&A, 336,925

Zijlstra, A. A., te Lintel Hekkert, P., Pottasch, S. R., et al. 1989, A\&A, 217, 157 (ZLP89)

Zijlstra, A. A., Gaylard, M. J., te Lintel Hekkert, P., et al. 1991, A\&A, 243, L9

Zuckerman, B., \& Dyck, H. M. 1986, ApJ, 311, 345 